\title{
Origins and Structures of Background Ions Produced by Fast-Atom Bombardment of Glycerol
}

\author{
Kenneth A. Caldwell and Michael L. Gross \\ Midwest Center for Mass Spectrometry, Department of Chemistry, University of Nebraska, Lincoln, Nebraska, \\ USA
}

\begin{abstract}
Accurate mass measurements were used to assign elemental compositions and tandem mass spectrometry was used to characterize the peak-at-every-mass background ions produced by kiloelectron-volt-particle bombardment of neat fast-atom bombardment matrices. The majority of the background ions observed in the mass spectrum of neat glycerol was identified. On the basis of the experiments with glycerol, a theory for the formation of background ions is presented. Results are discussed according to the chemical and physical changes that kiloelectron-volt-particle bombardment produces in the matrix. (f Am Soc Mass Spectrom 1994, 5, 72-91)
\end{abstract}

$\mathrm{B}$ ombardment by kiloelectron-volt particles is now an established approach for producing ions from nonvolatile materials. The report of shorl-lived signals from absorbed surface "contaminants" by early investigators [1-3] prompted Benninghoven et al. [4-6] to use a low flux of bombarding particles (approximately $10^{10}$ particles $/ \mathrm{s} \cdot \mathrm{cm}^{2}$ ) so that damaged areas of the surface would not be resampled by the primary ion beam - the static secondary ion mass spectrometry (SIMS) experiment [7]. Subsequently, Barber et al. [8-10] introduced the use of a liquid matrix and a fast-atom bombardment (FAB) source so that high-resolution magnetic sector instruments could be used as mass analyzers. Aberth et al. [11] and others [12, 13] showed later that the use of fast ions as the bombarding particles - the liquid SIMS experiment-produces results similar to those obtained by Barber et al. The incorporation of a liquid matrix into the SIMS experiment was a significant innovation [14], because it leads to longer-lived, more intense secondary ion beams, owing in part to the use of a higher flux of bombarding particles (approximately $10^{13}$ particles $/ \mathrm{s} \cdot \mathrm{cm}^{2}$ ).

A shortcoming of using a matrix is the concomitant production of matrix-derived ions. Most obvious are the protonated or deprotonated matrix molecule ions and their oligomers, for example, [(Matrix $+\mathrm{H})+$ nMatrix $]^{+}$, which are formed in the absence of added acid or base, even for aprotic matrices. Because these ions are easily identified, however, they seldom hinder FAB-based analyses. There is yet another, much larger, set of ions, the ubiquitous matrix-derived background

Address reprint requests to Professor Michael L. Gross, Department of Chemistry, University of Nebraska, Lincoln, NE 68588-0304. ions, which we denote as all ions other than the matrix molecule ion and its oligomers. Although this larger set of ions is oflen called "chemical noise," it was suggested [15] that this term is not appropriate. The ions do not produce noise in the conventional sense and should instead be referred to as background ions.

Matrix-derived background ions, which are detectable at every mass unit up to and beyond $2000 \mathrm{u}$, are the primary obstacle to low-level determinations by $\mathrm{FAB}$ and thwart advances in instrument design aimed at lowering detection limits. As the analyte concentration in the matrix is decreased, the background ions ultimately become what is analyzed [i.e., what is collisionally activated in a tandem mass spectrometry (MS/MS) experiment [16]]. A rule of thumb is that if the sample amount is $>1$ to $10 \mu \mathrm{g}$, then the abundance of the background ions is relatively low compared with those of the analyte. For analyses at the submicrogram level, however, the abundances of the analyte and the background ions become comparable. Moreover, for matrices that contain $\mathrm{C}, \mathrm{H}, \mathrm{O}$, and $\mathrm{N}$, the background ions usually cannot be resolved from analytes containing the same elements by using practical mass resolving power $[17,18]$. If the analyte ion can be resolved from the matrix background ions, then picogram analyses can be performed [17, 18]. Therefore, the primary motivation for the investigation reported here is to characterize background ions and determine their origin so that strategies to permit lowlevel determinations by FAB-MS and FAB-MS /MS [19] can be made more feasible.

The bombardment-induced chemistry leading to the formation of matrix-derived background ions can be abserved by bombarding a matrix to which no analyte 
has been added. Because the molar concentration (13.7 $M$ for glycerol) of the matrix is typically much greater than that of the analyte $\left(10^{-1}\right.$ to $\left.10^{-3} \mathrm{M}\right)$, the chemistry observed for the neat matrix is expected to occur when matrix and sample are mixed together. Even for analytes that are enriched at the surface, the atom beam still samples subsurface matrix molecules [20]. Moreover, as sample levels decrease, the chemistry of the neat matrix becomes increasingly important; Aberth and Burlingame [21] reported that for the liquid SIMS (LSIMS) analysis of $2.5 \mathrm{pmol}$ of methyl glucose polymer, "...the primary source of background signal at low sample levels appears to arise from the solvent matrix alone...."

We report the use of high-performance mass spectrometry tools for determining and characterizing background ions. The approach was to study bombardment-induced matrix chemistry in situ by using a tandem mass spectrometer capable of investigating ions at high resolving power; preliminary results were presented previously [18]. High-resolution peak matching was used to obtain the accurate mass and elemental composition of individual background ions. The fragmentations of mass-selected background ions were determined by using the MS/MS capability of the spectrometer.

\section{Experimental}

\section{Materials}

Glycerol (99.5\%) was obtained from Fisher Scientific (Fair Lawn, NJ). Approximately $50 \mathrm{~mL}$ was vacuum distilled. The forefraction was discarded, and only the heart of the distillate was collected. Solid dithiothreitol (DTT) and dithioerythreitol (DTE) were purchased from Aldrich Chemical Co. (Milwaukee, WI), mixed in the ratio 3:1 (DTT/DTE, $w / w$ ), and heated to $50^{\circ} \mathrm{C}$ to form a mixture, which, upon cooling, remained liquid at room temperature. Hydroxyethyl disulfide and thioglycerol were also purchased from Aldrich and used as received.

\section{Mass Spectrometry}

Three sectur mass spectrometer. Full scan spectra of source-produced ions and tandem mass spectra of individual ions were acquired by using a Kratos (Ramsey, NJ) MS50-TA [22] ( $\mathrm{E}_{1} \mathrm{BE}_{2}$ configuration) equipped with an Ion Tech (Middlesex, UK) FAB gun and a Kratos FAB ionization source. The atom gun was operated at $6 \mathrm{kV}$ with a discharge current of 1 to $2 \mathrm{~mA}$. The atom beam was oriented $90^{\circ}$ to the ion exit path, and argon was used exclusively as the FAB gas. Pressure within the source housing was typically $5 \times 10^{-6}$ torr.

Tables 1 and 2 of background ions were compiled in the following manner. Relative abundances of the major background jons were obtained by acquiring full scan spectra of the neat matrix by using the three sector mass spectrometer and the Kratos DS- 55 data system. Data acquisition was started after the probe had been in the atom beam for approximately $30 \mathrm{~s}$, and three scans were averaged to provide more precise relative abundances. Subsequently, both major and minor background ions were peak matched (at a resolution of at least 10,000) to known background ions or to matrix oligomer ions to obtain an accurate mass and formula of the unknown ion as well as its relative abundance. Fits to a chemically acceptable formula were usually within $5 \mathrm{ppm}$ of the theoretical mass. Because some of the background ions are proton-bound dimers of two saturated molecules, the number of hydrogen atoms exceeds what would be available for an intact, saturated, carbon-containing molecule. This violates valency rules in the fitting algorithm, and to avoid changing the algorithm, the program was tricked by subtracting the accurate mass of four $\mathrm{H}$ atoms from the experimental mass. The mass then corresponded to a possible molecular species, and the formula fitted to that experimental mass was simply the correct formula minus four $\mathrm{H}$ atoms.

Because the absolute abundance of many background ions was too low to allow a precise determination by using the DS-55 data system, the abundance of the unknown ion relative to that of a known matrix ion was recorded manually during the peak match experiment. By establishing the relative abundance of known matrix ions, the relative abundances of minor background ions were determined.

For all the MS/MS studies, the resolving power of the first-stage mass analyzer (MS-I) was 10,000 (10\% valley definition) or greater. For MS/MS experiments in which an odd-electron ion was selected, a resolving power of 17,000 to 20,000 was used to select the precursor ion. High resolving power was necessary to resolve at least in part the ${ }^{13} \mathrm{C}$-containing component of the even-electron ion one mass unit lower.

An electrostatic analyzer, $\mathrm{E}_{2}$, comprises MS-II. The MS/MS experiments were performed either without collision gas (metastable ion decompositions) or with helium, which was admitted to the collision cell localed afler the magnet in the third field-free region. Typically, 25\% beam reduction was used for the collision-activated dissociation (CAD) experiments.

Four sector tandem mass spectrometer. The first stage of a new-design VG Analytical ZAB-T prototype four-sector $\left(B_{1} E_{1} B_{2} E_{2}\right)$ mass spectrometer was also used in this study. This instrument was designed primarily for MS/MS experiments, and was briefly described [23]. The first stage mass analyzer consisted of a standard ZAB-SE of BE geometry. A VG LSIMS sour ze operated at $8 \mathrm{kV}$ was used with a $\mathrm{Cs}^{+}$ion gun operated at 25 $\mathrm{kV}$ to produce secondary ions (LSIMS). Data collection and work-up were accomplished by a Digital (Maynard, MA) 3100 M38 VAXstation computer running VG OPUS software. 
Table 1. Tabulated spectrum of glycerol positive background ions

\begin{tabular}{|c|c|c|c|c|}
\hline$m / z$ & Formula & Abundance & Comments and lon Origin & \\
\hline 13.00782 & $\mathrm{CH}$ & 0.01 & bond shredding, fragment of $\mathrm{CH}_{3}^{+}$ & \\
\hline 14.01565 & $\mathrm{CH}_{2}$ & 0.07 & $+\cdot$, bond shredding, fragment of $\mathrm{CH}_{3}{ }^{+}$ & \\
\hline 15.02347 & $\mathrm{CH}_{3}$ & 0.8 & fragment of: Gly ${ }^{\ddagger},\left[\mathrm{Gly}+\mathrm{H}^{+}\right.$ & \\
\hline 15.99491 & $\mathbf{O}$ & 0.003 & $+\cdot$, bond shredding & \\
\hline 17.00274 & $\mathrm{OH}$ & 0.02 & heterolytic bond cleavage & \\
\hline 18.01056 & $\mathrm{H}_{2} \mathrm{O}$ & 0.2 & $+\cdot$, fragment of Glyt & \\
\hline 19.01839 & $\mathrm{H}_{3} \mathrm{O}$ & 6.1 & hydronium ion, TD, [Gly $\left.-G_{74}+\mathrm{H}\right]$, fragment of Gly: & \\
\hline 26.01565 & $\mathrm{C}_{2} \mathrm{H}_{2}$ & 0.1 & $+\bullet$, fragment of Glyt & \\
\hline 27.02347 & $\mathrm{C}_{2} \mathrm{H}_{3}$ & 1.2 & fragment of: $\mathrm{Gly} t,[\mathrm{Gly}+\mathrm{H}]^{+},\left[\mathrm{Gly}-\mathrm{H}_{2} \mathrm{O}+\mathrm{H}\right]^{+}$ & \\
\hline 28.03130 & $\mathrm{C}_{2} \mathrm{H}_{4}$ & 0.9 & $+\cdot$, fragment of Gly: & \\
\hline 27.99491 & $\mathrm{CO}$ & 0.02 & $+\cdot$, fragment ion & \\
\hline 29.03912 & $\mathrm{C}_{2} \mathrm{H}_{3}$ & 4.6 & fragment of Gly‡ & \\
\hline 29.00274 & $\mathrm{CHO}$ & 1.8 & fragment of: $\mathrm{Gly} y^{\ddagger},[\mathrm{Gly}+\mathrm{H}]^{+},\left[\mathrm{Gly}-\mathrm{H}_{2} \mathrm{O}+\mathrm{H}\right]^{+}$ & \\
\hline 30.01056 & $\mathrm{CH}_{2} \mathrm{O}$ & 0.1 & $+\cdot$, fragment of Glyt & \\
\hline 30.04248 & $\mathrm{C}^{13} \mathrm{CH}_{5}$ & 0.1 & fragment of Gly: & \\
\hline 30.04695 & $\mathrm{C}_{2} \mathrm{H}_{6}$ & 0.1 & $+\cdot$, fragment of Glyt & \\
\hline 31.01839 & $\mathrm{CH}_{3} \mathrm{O}$ & 4.5 & TD, fragment of: $[\mathrm{Gly}+\mathrm{H}]^{+},\left[\mathrm{Gly}-\mathrm{H}_{2} \mathrm{O}+\mathrm{H}\right]^{+}$ & \\
\hline 33.03404 & $\mathrm{CII}_{5} \mathrm{O}$ & 0.06 & sal'd, TD, $\left[G_{32}+\mathrm{H}\right]^{+}$, fragment of Gly๋ & \\
\hline 39.02347 & $\mathrm{C}_{3} \mathrm{H}_{3}$ & 0.5 & fragment of Gly: & \\
\hline 41.03912 & $\mathrm{C}_{3} \mathrm{H}_{5}$ & 0.6 & {$\left[G_{76}-\mathrm{H}_{2} \mathrm{O}-\mathrm{C}_{2} \mathrm{H}_{4}+\mathrm{H}\right]^{+}$} & \\
\hline 42.01056 & $\mathrm{C}_{2} \mathrm{H}_{2} \mathrm{O}$ & 0.4 & $+\bullet$, fragment of: $\mathrm{Gly}:\left[G_{90}+H\right]^{+}$ & \\
\hline 42.04695 & $\mathrm{C}_{3} \mathrm{H}_{6}$ & 0.1 & $+\bullet$ & \\
\hline 43.01839 & $\mathrm{C}_{2} \mathrm{H}_{3} \mathrm{O}$ & 2.6 & fragment of: Glyt, $\left[G_{90}+\mathrm{H}\right]^{+}$ & \\
\hline 43.05477 & $\mathrm{C}_{3} \mathrm{H}$ & 0.1 & fragment of various species higher in mass & \\
\hline 44.02621 & $\mathrm{C}_{2} \mathrm{H}_{4} \mathrm{O}$ & 1.1 & $+\cdot$ fragment of Glyt & \\
\hline 45.03404 & $\mathrm{C}_{2} \mathrm{H}_{3} \mathrm{O}$ & 15 & $\mathrm{TD}$, fragment of: Glyt, $[\mathrm{Gly}+\mathrm{H}]^{+}$ & \\
\hline 46.03739 & $\mathrm{C}^{13} \mathrm{CH}_{5} \mathrm{O}$ & 0.3 & TD, fragment of: Glyt, $[\mathrm{Gly}+\mathrm{H}]^{+}$ & \\
\hline 47.01330 & $\mathrm{CH}_{3} \mathrm{O}_{2}$ & 1.1 & {$\left[\mathrm{Gly}-\mathrm{H}_{2} \mathrm{O}-\mathrm{C}_{2} \mathrm{H}_{4}+\mathrm{H}\right]^{+}$} & \\
\hline 47.04969 & $\mathrm{C}_{2} \mathrm{H}_{3} \mathrm{O}$ & 0.2 & sat'd, $\left[\mathrm{Gly}-\mathrm{H}_{2} \mathrm{O}-\mathrm{CO}+\mathrm{H}\right]^{+}$ & \\
\hline 49.02895 & $\mathrm{CH}_{5} \mathrm{O}_{2}$ & 0.1 & {$\left[G_{76}-\mathrm{C}_{2} \mathrm{H}_{4}+\mathrm{H}\right]^{+}$} & \\
\hline 55.01839 & $\mathrm{C}_{3} \mathrm{H}_{3} \mathrm{O}$ & 0.8 & fragment of Gly* and species higher in mass & \\
\hline 55.05477 & $\mathrm{C}_{4} \mathrm{II}_{7}$ & 0.1 & fragment of Gly aggregate & \\
\hline 56.02621 & $\mathrm{C}_{3} \mathrm{H}_{4} \mathrm{O}$ & 1.6 & $+\bullet$ fragment of Glyt & \\
\hline 57.03404 & $\mathrm{C}_{3} \mathrm{H}_{3} \mathrm{O}$ & 16.0 & TD, $\left[\mathrm{Gly}-2 \mathrm{H}_{2} \mathrm{O}+\mathrm{H}\right]^{+}$, fragment of Glyt, metastable: $29\left(-\mathrm{C}_{2} \mathrm{H}_{4}\right.$ and/or $\left.\mathrm{CO}\right)$ & \\
\hline 58.04186 & $\mathrm{C}_{3} \mathrm{H}_{6} \mathrm{O}$ & 0.6 & + & \\
\hline 59.04969 & $\mathrm{C}_{3} \mathrm{H}_{7} \mathrm{O}$ & 0.3 & {$\left[G_{76}-\mathrm{H}_{2} \mathrm{O}+\mathrm{H}\right]^{+}$} & \\
\hline 60.02113 & $\mathrm{C}_{2} \mathrm{H}_{4} \mathrm{O}_{2}$ & 0.6 & $+\cdot$, fragment of Gly: & \\
\hline 61.02895 & $\mathrm{H}_{2} \mathrm{C}_{2} \mathrm{H}_{3} \mathrm{O}_{2}$ & 2.1 & $\mathrm{TD},[\mathrm{Gly}+\mathrm{H}-\mathrm{MeOH}]^{+}$and $\left[G_{\infty}+\mathrm{H}\right]^{+}$, analogous $\mathrm{Cs}_{2} \mathrm{C}_{2} \mathrm{H}_{3} \mathrm{O}_{2}$ ion in Gly/CsI matrix & \\
\hline 62.03678 & $\mathrm{C}_{2} \mathrm{H}_{6} \mathrm{O}_{2}$ & 0.04 & $+\cdot$, fragment of: Gly $\ddagger$, $[\mathrm{Gly}+\mathrm{H}]^{+}$ & \\
\hline 63.04460 & $\mathrm{C}_{2} \mathrm{H}_{7} \mathrm{O}_{2}$ & 0.03 & {$\left[G_{62}+\mathrm{H}\right]^{+}$} & \\
\hline 67.05477 & $\mathrm{C}_{5} \mathrm{H}_{7}$ & 0.09 & & \\
\hline 69.03404 & $\mathrm{C}_{4} \mathrm{H}_{5} \mathrm{O}$ & 0.4 & {$\left[G_{192}-3 \mathrm{H}_{2} \mathrm{O}-\mathrm{H}_{2} \mathrm{CO}+\mathrm{H}\right]^{+},\left[G_{122}-3 \mathrm{H}_{2} \mathrm{O}+\mathrm{H}\right]^{+}$} & \\
\hline 70.04186 & $\mathrm{C}_{4} \mathrm{H}_{6} \mathrm{O}$ & 0.1 & $+*$ fragment of a Gly aggregate species & \\
\hline 71.04969 & $\mathrm{C}_{4} \mathrm{H}_{7} \mathrm{O}$ & 0.4 & {$\left[G_{106}-2 \mathrm{H}_{2} \mathrm{O}+\mathrm{H}\right]^{+},\left[G_{152}-3 \mathrm{H}_{2} \mathrm{O}-\mathrm{CO}+\mathrm{H}\right]^{+}$} & (contimued) \\
\hline
\end{tabular}

For aggregate species, braces are used as a guide to indicate the formal composition of the ion, but isomeric structures may also contribute to the ion population. Ion origins are based on tandem mass spectra and elemental composirions. See text.

+ = radical cation.

TD = this ion that may also arise from protonation of a thermal decomposition product.

sat'd = saturated species or a proton bound oligomer corrposed of an unsaturated molecule and one or more glycerol molecules.

Metastable or CAD = The losses, product ions, or the mass-to-charge ratio of the product ions that result from metastable ion or CA decompositions. The ions are listed by decreasing abundance, and... indicates the omission of ions of intermediate abundance. 
Table 1-(contimued)

\begin{tabular}{|c|c|c|c|}
\hline $\mathrm{m} / \mathrm{z}$ & Formula & Abundance & Comments and lon Origin \\
\hline 71.01330 & $\mathrm{C}_{3} \mathrm{H}_{3} \mathrm{O}_{2}$ & 0.2 & {$\left[G_{152}-3 \mathrm{H}_{2} \mathrm{O}-\mathrm{C}_{2} \mathrm{H}_{4}+\mathrm{H}\right]^{+}$} \\
\hline 72.02113 & $\mathrm{C}_{3} \mathrm{H}_{4} \mathrm{O}_{2}$ & 0.3 & $+*\left[G_{90}-\mathrm{H}_{2} \mathrm{O}\right] t$ \\
\hline 73.02895 & $\mathrm{C}_{3} \mathrm{II}_{5} \mathrm{O}_{2}$ & 1.6 & {$\left[G_{90}-\mathrm{H}_{2} \mathrm{O}+\mathbf{H}\right]^{+}$} \\
\hline 74.03678 & $\mathrm{C}_{3} \mathrm{H}_{6} \mathrm{O}_{2}$ & 3.3 & $+\cdot$, fragment of: $\mathrm{Gly}+,[\mathrm{Gly}+\mathrm{H}]^{+}$ \\
\hline 75.04460 & $\mathrm{C}_{3} \mathrm{H}_{7} \mathrm{O}_{2}$ & 23 & $\mathrm{TD},\left[\mathrm{Gly}+\mathrm{H}-\mathrm{H}_{2} \mathrm{O}\right]$ \\
\hline 77.06025 & $\mathrm{C}_{3} \mathrm{H}_{9} \mathrm{O}_{2}$ & 0.09 & $\operatorname{sal}^{*} \mathrm{~d},\left[G_{76}+\mathrm{H}\right]^{+}$ \\
\hline 81.03404 & $\mathrm{C}_{9} \mathrm{H}_{3} \mathrm{O}$ & 0.3 & {$\left[G_{132}-4 \mathrm{H}_{2} \mathrm{O}+\mathrm{H}\right]^{+},\left[G_{182}-4 \mathrm{H}_{2} \mathrm{O}-\mathrm{H}_{2} \mathrm{CO}+\mathrm{H}\right]^{+}$} \\
\hline 81.07042 & $\mathrm{C}_{6} \mathrm{H}_{9}$ & 0.2 & \\
\hline 83.04969 & $\mathrm{C}_{3} \mathrm{H}_{3} \mathrm{O}$ & 0.2 & {$\left[G_{136}-3 \mathrm{H}_{3} \mathrm{O}+\mathrm{H}\right]^{*}$} \\
\hline 85.02895 & $\mathrm{C}_{4} \mathrm{H}_{5} \mathrm{O}_{2}$ & 0.2 & {$\left[G_{132}-2 \mathrm{H}_{2} \mathrm{O}-\mathrm{CH}_{3} \mathrm{OH}-\mathrm{H}_{2} \mathrm{CO}+\mathrm{H}\right]^{+}$} \\
\hline 86.03678 & $\mathrm{C}_{4} \mathrm{H}_{6} \mathrm{O}_{2}$ & 0.2 & + \\
\hline 87.04460 & $\mathrm{C}_{4} \mathrm{H}_{7} \mathrm{O}_{2}$ & 0.3 & {$\left[G_{127}-2 \mathrm{H}_{2} \mathrm{O}+\mathrm{H}\right]^{+}$} \\
\hline 89.06025 & $\mathrm{C}_{4} \mathrm{H}_{4} \mathrm{O}_{2}$ & 0.2 & {$\left[\mathrm{G}_{106}-\mathrm{H}_{2} \mathrm{O}+\mathrm{H}\right]^{+}$} \\
\hline 89.03912 & $\mathrm{C}_{3} \mathrm{H}_{5}$ & $<0.001$ & abundance increases with time in beam \\
\hline 90.03169 & $\mathrm{C}_{3} \mathrm{H}_{6} \mathrm{O}_{3}$ & 0.7 & $+\bullet\left[G_{90}\right] \pm \mathrm{CAD}_{i}-\mathrm{H}_{2} \mathrm{O}$ \\
\hline 91.03952 & $\mathrm{C}_{3} \mathrm{H}_{7} \mathrm{O}_{3}$ & 0.4 & {$\left[G_{90}+\mathrm{H}\right] \mathrm{CAD}:-\mathrm{H}_{2} \mathrm{CO}_{r}-\mathrm{H}_{2} \mathrm{O}$} \\
\hline 92.04287 & $\mathrm{C}_{2}{ }^{13} \mathrm{CH}_{7} \mathrm{O}_{3}$ & 0.04 & {$\left[G_{90}+H^{+}\right.$} \\
\hline 92.03594 & $\mathrm{C}_{3} \mathrm{H}_{6} \mathrm{O}_{2}{ }^{18} \mathrm{O}$ & 0.01 & $+\cdot\left[G_{90}\right] \pm$ \\
\hline 92.04734 & $\mathrm{C}_{3} \mathrm{H}_{8} \mathrm{O}_{3}$ & 0.01 & $+\cdot$, Gly: \\
\hline 93.05517 & $\mathrm{C}_{3} \mathrm{H}_{9} \mathrm{O}_{3}$ & 100.00 & {$[\mathrm{Gly}+\mathrm{H}]^{+} \mathrm{CAD}:-\mathrm{H}_{2} \mathrm{O},-2 \mathrm{H}_{2} \mathrm{O},-\left(\mathrm{II}_{2} \mathrm{O}+\mathrm{II}_{2} \mathrm{CO}\right),-\mathrm{Cl}_{3} \mathrm{OH}, \ldots-\mathrm{OH}_{2} \mathrm{H}_{3} \mathrm{O}^{+}, \mathrm{CH}_{3}^{+}$} \\
\hline 94.05852 & $\mathrm{C}_{2}{ }^{13} \mathrm{CH}_{9} \mathrm{O}_{3}$ & 3.6 & {$[\mathrm{Gly}+\mathrm{H}]^{+}$} \\
\hline 95.05941 & $\mathrm{C}_{3} \mathrm{H}_{9} \mathrm{O}_{2}{ }^{1 *} \mathrm{O}$ & 0.6 & {$[\mathrm{Gly}+\mathrm{H}]^{+}$} \\
\hline 95.04969 & $\mathrm{C}_{6} \mathrm{H}_{7} \mathrm{O}$ & 0.1 & {$\left[G_{166}-4 \mathrm{H}_{2} \mathrm{O}+\mathrm{H}\right]^{+}$} \\
\hline 97.02895 & $\mathrm{C}_{9} \mathrm{H}_{5} \mathrm{O}_{2}$ & 0.05 & {$\left[G_{182}-3 \mathrm{H}_{2} \mathrm{O}-\mathrm{CH}_{3} \mathrm{OH}+\mathrm{H}\right]^{+}$} \\
\hline 99.04460 & $\mathrm{C}_{3} \mathrm{H}_{7} \mathrm{O}_{2}$ & 0.1 & {$\left[G_{152}-3 \mathrm{H}_{2} \mathrm{O}+\mathrm{H}\right]^{+}$} \\
\hline 101.06025 & $\mathrm{C}_{5} \mathrm{H}_{9} \mathrm{O}_{2}$ & 0.2 & {$\left[G_{136}-2 \mathrm{H}_{2} \mathrm{O}+\mathrm{H}\right]^{+}$} \\
\hline 103.03952 & $\mathrm{C}_{4} \mathrm{H}_{7} \mathrm{O}_{3}$ & 0.1 & {$\left[G_{152}-\mathrm{H}_{2} \mathrm{O}-\mathrm{CH}_{3} \mathrm{OH}+\mathrm{H}\right]^{+}$} \\
\hline 105.05517 & $\mathrm{C}_{4} \mathrm{H}_{9} \mathrm{O}_{3}$ & 0.1 & {$\left[G_{122}-\mathrm{H}_{2} \mathrm{O}+\mathrm{H}\right]^{+} \mathrm{CAD}:-\mathrm{H}_{2} \mathrm{O},-\left(\mathrm{H}_{2} \mathrm{O}+\mathrm{H}_{2} \mathrm{CO}\right),-\mathrm{H}_{2} \mathrm{CO}$} \\
\hline 107.07082 & $\mathrm{C}_{4} \mathrm{H}_{11} \mathrm{O}_{3}$ & 0.5 & sat $^{\prime} \mathrm{d},\left[\mathrm{G}_{10 \leqslant}+\mathrm{H}\right]^{+} \mathrm{CAD} ;-\mathrm{H}_{2} \mathrm{O},-2 \mathrm{H}_{2} \mathrm{O}, \ldots-\mathrm{CH}_{3} \mathrm{OH}$ \\
\hline 111.04460 & $\mathrm{C}_{6} \mathrm{H}_{7} \mathrm{O}_{2}$ & 0.06 & {$\left[G_{182}-4 \mathrm{H}_{2} \mathrm{O}+\mathrm{H}\right]^{+} \mathrm{CAD}:-\mathrm{CO}$ and $/ \mathrm{or}-\mathrm{C}_{2} \mathrm{H}_{4},-\mathrm{H}_{2} \mathrm{CO}$} \\
\hline 111.06573 & $\mathrm{C}_{3} \mathrm{H}_{11} \mathrm{O}_{4}$ & 0.01 & sat'd, Gly aggregate: $\left[\mathrm{H}_{2} \mathrm{O}+\mathrm{Gly}+\mathrm{H}\right]^{+}$metastable: $-\mathrm{H}_{2} \mathrm{O}$ \\
\hline 113.06025 & $\mathrm{C}_{6} \mathrm{H}_{9} \mathrm{O}_{2}$ & 0.2 & {$\left[G_{166}-3 \mathrm{H}_{2} \mathrm{O}+\mathrm{H}\right]^{+} \mathrm{CAD}:-\mathrm{H}_{2} \mathrm{O},-\mathrm{CH}_{2},-\mathrm{CO}$ and/or $-\mathrm{C}_{2} \mathrm{H}_{4}$} \\
\hline 115.03952 & $\mathrm{C}_{3} \mathrm{H}_{7} \mathrm{O}_{3}$ & 0.1 & {$\left[G_{182}-2 \mathrm{H}_{2} \mathrm{O}-\mathrm{CH}_{3} \mathrm{OH}+\mathrm{H}\right]^{+} \mathrm{CAD}:-\mathrm{H}_{2} \mathrm{O},-\mathrm{H}_{2} \mathrm{CO}, \ldots-\mathrm{CH}_{2}$} \\
\hline 115.07590 & $\mathrm{C}_{6} \mathrm{H}_{11} \mathrm{O}_{2}$ & 0.1 & {$\left[\left\{G_{76}-\mathrm{H}_{2} \mathrm{O}\right\}+G_{56}+\mathrm{H}\right]^{+}$} \\
\hline 117.05517 & $\mathrm{C}_{9} \mathrm{H}_{9} \mathrm{O}_{3}$ & 0.5 & {$\left[G_{152}-2 \mathrm{H}_{2} \mathrm{O}+\mathrm{H}\right]^{+} \mathrm{CAD}:-\mathrm{H}_{2} \mathrm{O}, 57,-\left(\mathrm{H}_{2} \mathrm{O}+\mathrm{H}_{2} \mathrm{CO}\right)$} \\
\hline 117.07042 & $\mathrm{C}_{9} \mathrm{H}_{9}$ & $<0.001$ & abundance increases with time in beam \\
\hline 119.07082 & $\mathrm{C}_{5} \mathrm{H}_{11} \mathrm{O}_{3}$ & 0.2 & {$\left[G_{136}-\mathrm{H}_{2} \mathrm{O}+\mathrm{H}\right]^{+} \mathrm{CAD}:-\mathrm{H}_{2} \mathrm{O}, 75,-\mathrm{CH}_{2}$} \\
\hline 123.06573 & $\mathrm{C}_{4} \mathrm{H}_{11} \mathrm{O}_{4}$ & 0.6 & sat'd, $\left[G_{122}+H^{+} \mathrm{CAD}:-\mathrm{H}_{2} \mathrm{O},-2 \mathrm{H}_{2} \mathrm{O}\right.$ \\
\hline 127.07590 & $\mathrm{C}_{7} \mathrm{H}_{11} \mathrm{O}_{2}$ & 0.04 & fragment of $\left.\left[\mid G_{106}-2 \mathrm{H}_{2} \mathrm{O}\right\}+\mathrm{Gly}+\mathrm{H}\right]^{+}$ \\
\hline 129.05517 & $\mathrm{C}_{6} \mathrm{H}_{9} \mathrm{O}_{3}$ & 0.5 & {$\left[G_{182}-3 \mathrm{H}_{2} \mathrm{O}+\mathrm{H}^{+} \mathrm{CAD}:-\mathrm{H}_{2} \mathrm{O},-\mathrm{H}_{2} \mathrm{CO}\right.$} \\
\hline 131.07082 & $\mathrm{C}_{6} \mathrm{H}_{11} \mathrm{O}_{3}$ & 0.7 & $\begin{array}{l}{\left[G_{166}-2 \mathrm{H}_{2} \mathrm{O}+\mathrm{H}\right]^{+},\left[\left(G_{56}+\mathrm{Gly}+\mathrm{H}\right)-\mathrm{H}_{2} \mathrm{O}\right]^{+},\left[G_{56}+G_{74}+\mathrm{H}\right]^{+} \text {metastable: }-\mathrm{H}_{2} \mathrm{O},-\mathrm{CH}_{2},-\mathrm{H}_{2} \mathrm{CO}} \\
{\left[G_{74}+\mathrm{H}\right]^{+} \mathrm{CAD}:-\mathrm{H}_{2} \mathrm{O},-\mathrm{H}_{2} \mathrm{CO}}\end{array}$ \\
\hline 133.05008 & $\mathrm{C}_{5} \mathrm{H}_{9} \mathrm{O}_{4}$ & 0.07 & fragment of $\left[G_{182}-\mathrm{H}_{2} \mathrm{O}-\mathrm{CH}_{3} \mathrm{OH}+\mathrm{H}\right]^{+}$ \\
\hline 133.08646 & $\mathrm{C}_{6} \mathrm{H}_{13} \mathrm{O}_{3}$ & 0.06 & {$\left[\left\{G_{76}-\mathrm{H}_{2} \mathrm{O}\right\}+G_{74}+\mathrm{H}\right]^{+}$, fragment of $\left[\left\{G_{106}-2 \mathrm{H}_{2} \mathrm{O}\right\}+\mathrm{Gly}+\mathrm{H}\right]^{+} \mathrm{CAD}:-\mathrm{H}_{2} \mathrm{O},\left[G_{74}+\mathrm{H}\right]^{+}$} \\
\hline 134.05791 & $\mathrm{C}_{5} \mathrm{H}_{10} \mathrm{O}_{4}$ & 0.03 & $+\cdots\left[\mathrm{C}_{2} \mathrm{H}_{2} \mathrm{O}+\mathrm{Gly}\right]^{t}$, metastable: $-\mathrm{H}_{2} \mathrm{O},-\mathrm{CH}_{3}$ \\
\hline 135.06573 & $\mathrm{C}_{5} \mathrm{H}_{11} \mathrm{O}_{4}$ & 0.2 & {$\left[G_{152}-\mathrm{H}_{2} \mathrm{O}+\mathrm{H}\right]^{+}$and $\left[G_{60}+G_{74}+\mathrm{H}\right]^{+} \mathrm{CAD}:-\mathrm{H}_{2} \mathrm{O},-\left(\mathrm{H}_{2} \mathrm{O}-G_{60}\right),-G_{60}$} \\
\hline 136.07356 & $\mathrm{C}_{5} \mathrm{H}_{12} \mathrm{O}_{4}$ & $<0.1$ & $+\bullet$ Gly aggregate: $\left[\mathrm{C}_{2} \mathrm{H}_{4} \mathrm{O}+\left.\mathrm{Gly}\right|^{t} \mathrm{CAD}:-\mathrm{H}_{2} \mathrm{O},[\mathrm{Gly}+\mathrm{H}]^{+}\right.$ \\
\hline 137.08138 & $\mathrm{C}_{5} \mathrm{H}_{13} \mathrm{O}_{4}$ & 0.4 & sat'd. $\left[G_{61}-G_{75}+\mathrm{H}\right\rceil^{+}$i. e. $\left\lceil G_{136}+\mathrm{H}\right]^{+} \mathrm{CAD}:-\mathrm{H}_{2} \mathrm{O},-2 \mathrm{H}_{2} \mathrm{O}, 57,83,75$ \\
\hline 143.07082 & $\mathrm{C}_{7} \mathrm{H}_{11} \mathrm{O}_{3}$ & 0.1 & Gly aggregate \\
\hline 145.08645 & $\mathrm{C}_{7} \mathrm{H}_{13} \mathrm{O}_{3}$ & 0.04 & fragment of $\left.\left[1 G_{105}-2 \mathrm{H}_{2} \mathrm{O}\right]+\mathrm{Gly}+\mathrm{H}\right]^{+}$ \\
\hline 147.06573 & $\mathrm{C}_{6} \mathrm{H}_{11} \mathrm{O}_{4}$ & 0.4 & {$\left[G_{182}-2 \mathrm{H}_{2} \mathrm{O}+\mathrm{H}\right]^{+}$metastable: $-\mathrm{H}_{2} \mathrm{O},-\mathrm{H}_{2} \mathrm{CO},-\left(\mathrm{H}_{2} \mathrm{O}+\mathrm{H}_{2} \mathrm{CO}\right)$} \\
\hline
\end{tabular}


Table 1-(continued)

\begin{tabular}{|c|c|c|c|}
\hline $\mathrm{m} / 2$ & Formula & Abundance & Comments and lon Origin \\
\hline 149.08138 & $\mathrm{C}_{6} \mathrm{H}_{13} \mathrm{O}_{4}$ & 1.2 & $\begin{array}{l}\mathrm{TD},\left[G_{166}-\mathrm{H}_{2} \mathrm{O}+\mathrm{H}\right]^{+},\left[G_{56}+\mathrm{Gly}+\mathrm{H}\right]^{+},\left[\left(G_{74}+\mathrm{Gly}+\mathrm{H}\right)-\mathrm{H}_{2} \mathrm{O}\right]^{+},\left[2 G_{74}+\mathrm{H}\right]^{+} \mathrm{CAD}:-\mathrm{H}_{2} \mathrm{O},-G_{74} \\
{\left[G_{74}+\mathrm{H}\right]^{+},-\mathrm{Gly}}\end{array}$ \\
\hline 151.09703 & $\mathrm{C}_{6} \mathrm{H}_{15} \mathrm{O}_{4}$ & 0.3 & $\begin{array}{l}\text { sat'd, }\left[G_{76}+G_{74}+\mathrm{H}\right]^{+}, \text {Gly aggregate: }\left[\left\{G_{76}-\mathrm{H}_{2} \mathrm{O}\right\}+\mathrm{Gly}+\mathrm{H}\right]^{+} \mathrm{CAD}:-\mathrm{H}_{2} \mathrm{O},-2 \mathrm{H}_{2} \mathrm{O},\left[G_{74}+\mathrm{H}\right]^{+} \\
{\left[G_{76}-\mathrm{H}_{2} \mathrm{O}+\mathrm{H}\right]^{+},-\left(G_{76}-\mathrm{H}_{2} \mathrm{O}\right)}\end{array}$ \\
\hline 152.06848 & $\mathrm{C}_{5} \mathrm{H}_{12} \mathrm{O}_{5}$ & 0.2 & $+\cdot$, Gly aggregate: $\left[\mathrm{C}_{2} \mathrm{H}_{4} \mathrm{O}_{2}+\mathrm{Gly}\right]: \mathrm{CAD}:[\mathrm{Gly}+\mathrm{H}]^{4}, \mathrm{C}_{2} \mathrm{H}_{4} \mathrm{O}_{2} *$ \\
\hline 153.07630 & $\mathrm{C}_{5} \mathrm{H}_{13} \mathrm{O}_{s}$ & 0.6 & sat'd. $\left[G_{152}+\mathrm{H}\right]^{+} \mathrm{CAD}:-\mathrm{H}_{2} \mathrm{O},-2 \mathrm{H}_{2} \mathrm{O},-3 \mathrm{H}_{3} \mathrm{O}$ \\
\hline 155.07082 & $\mathrm{C}_{\mathrm{B}} \mathrm{H}_{11} \mathrm{O}_{3}$ & 0.03 & fragment of $\left[\left\{G_{152}-3 \mathrm{H}_{2} \mathrm{O}\right\}+\mathrm{Gly}+\mathrm{H}\right]^{+}$ \\
\hline 161.08138 & $\mathrm{C}_{7} \mathrm{H}_{19} \mathrm{O}_{4}$ & 0.1 & $\begin{array}{l}\text { Gly aggregate: }\left[\left\{G_{152}-3 \mathrm{H}_{2} \mathrm{O}-\mathrm{H}_{2} \mathrm{CO}\right\}+\mathrm{Gly}+\mathrm{H}\right]^{+} \text {and }\left[\left[G_{122}-3 \mathrm{H}_{2} \mathrm{O}+\mathrm{Gly}+\mathrm{H}\right]^{+} \text {, fragment of }\right. \\
{\left[\left\{G_{122}-\mathrm{H}_{2} \mathrm{O}\right]+\mathrm{Gly}+\mathrm{H}\right]^{+}}\end{array}$ \\
\hline 163.06065 & $\mathrm{C}_{6} \mathrm{H}_{11} \mathrm{O}_{5}$ & 0.09 & \\
\hline 163.09703 & $\mathrm{C}_{\mathrm{T}} \mathrm{H}_{1} \mathrm{SO}_{4}$ & 0.004 & Gly aggregate: $\left[\left\{G_{10 \%}-2 \mathrm{H}_{2} \mathrm{O}\right]+\mathrm{Gly}+\mathrm{H}\right]^{+} \mathrm{CAD}:-\mathrm{H}_{2} \mathrm{O},-2 \mathrm{H}_{2} \mathrm{O},-\mathrm{Gly}$ \\
\hline 165.07630 & $\mathrm{C}_{6} \mathrm{H}_{13} \mathrm{O}_{3}$ & 0.4 & {$\left[G_{\text {lax }}-\mathrm{H}_{3} \mathrm{O}+\mathrm{H}\right]^{+},\left[\left[G_{90}-\mathrm{H}_{z} \mathrm{O}\right]+\mathrm{Gly}+\mathrm{H}\right]^{+} \mathrm{CAD}:-\mathrm{H}_{z} \mathrm{O},-\mathrm{Gly}_{1}[\mathrm{Gly}+\mathrm{H}]^{+}$} \\
\hline 166.08412 & $\mathrm{C}_{6} \mathrm{H}_{14} \mathrm{O}_{5}$ & 0.1 &,+- Gly aggregate: $\left[\mathrm{C}_{3} \mathrm{H}_{6} \mathrm{O}_{2}+\mathrm{Gly}\right] \pm \mathrm{CAD}:[\mathrm{Gly}+\mathrm{H}]^{+}, \mathrm{C}_{3} \mathrm{H}_{6} \mathrm{O}_{2}:$ \\
\hline 167.09195 & $\mathrm{C}_{6} \mathrm{H}_{15} \mathrm{O}_{5}$ & 1.3 & sal'd, $\left[G_{166}+\mathrm{H}\right]^{+}$and/or $\left[G_{74}+\mathrm{Gly}+\mathrm{H}\right]^{+} \mathrm{CAD}:-\mathrm{H}_{2} \mathrm{O},-2 \mathrm{H}_{2} \mathrm{O}_{7}-3 \mathrm{H}_{2} \mathrm{O},\left[G_{74}+\mathrm{H}\right]^{+},[\mathrm{Gly}+\mathrm{H}]^{+}$ \\
\hline 169.10760 & $\mathrm{C}_{6} \mathrm{H}_{17} \mathrm{O}_{5}$ & 0.1 & sat'd, Gly aggregate: $\left[\left(G_{76}+\mathrm{H}\right)+\mathrm{Gly}\right]^{+} \mathrm{CAD}:[\mathrm{Gly}+\mathrm{H}]^{+},\left[G_{76}+\mathrm{H}\right]^{+}$ \\
\hline 173.08138 & $\mathrm{C}_{3} \mathrm{H}_{13} \mathrm{O}_{4}$ & 0.1 & $\begin{array}{l}\text { Gly aggregate: }\left[\left[G_{182}-4 \mathrm{H}_{2} \mathrm{O}-\mathrm{H}_{2} \mathrm{CO}\right\}+\mathrm{Gly}+\mathrm{H}\right]^{+} \text {and } \\
\left.\left[\left[G_{152}-4 \mathrm{H}_{2} \mathrm{O}\right\}+\mathrm{Gly}+\mathrm{H}\right]^{+} \text {, fragment of: }\left[\left\{G_{182}-3 \mathrm{H}_{2} \mathrm{O}+\mathrm{H}\right\}+\mathrm{G}\right] \mathrm{y}\right]^{+} \text {and }\left[\left\{G_{152}-2 \mathrm{H}_{2} \mathrm{O}\right\}+\mathrm{Gly}+\mathrm{H}\right]^{+}\end{array}$ \\
\hline 175.09703 & $\mathrm{C}_{8} \mathrm{H}_{15} \mathrm{O}_{4}$ & 0.03 & Gly aggregate: $\left.\left[\left\{G_{136}-3 \mathrm{H}_{2} \mathrm{O}\right\}+\mathrm{Gl}\right\} \mathrm{y}+\mathrm{H}\right]^{+}$ \\
\hline 177.06065 & $\mathrm{C}_{7} \mathrm{H}_{13} \mathrm{O}_{5}$ & 0.07 & Gly aggregate: $\left[\left[G_{182}-2 \mathrm{H}_{2} \mathrm{O}-\mathrm{CH}_{3} \mathrm{OH}-\mathrm{H}_{2} \mathrm{CO}\right]+\mathrm{Gly}+\mathrm{H}\right]^{+}$ \\
\hline 179.09195 & $\mathrm{C}_{7} \mathrm{H}_{15} \mathrm{O}_{5}$ & 0.08 & Gly aggregate, fragment of $\left[\left\{G_{\mathfrak{i z 2}}-\mathrm{H}_{2} \mathrm{O}\right\}+\mathrm{Gly}+\mathrm{H}\right]^{+}$ \\
\hline 181.10760 & $\mathrm{C}_{7} \mathrm{H}_{17} \mathrm{O}_{5}$ & 0.08 & sat'd, Gly aggregate: $\left.\left[\mid G_{106}-\mathrm{H}_{2} \mathrm{O}\right\}+\mathrm{Gly}+\mathrm{H}\right]^{+}$ \\
\hline 182.07904 & $\mathrm{C}_{6} \mathrm{H}_{14} \mathrm{O}_{6}$ & 0.2 & $+\bullet$, Gly aggregate: $\left[\mathrm{C}_{3} \mathrm{H}_{5} \mathrm{O}_{3}+\mathrm{Gly}\right]^{t}$, metastable: $\mathrm{C}_{3} \mathrm{H}_{5} \mathrm{O}_{3}{ }^{\ddagger},[\mathrm{Gly}+\mathrm{H}]^{+}$ \\
\hline 183.08686 & $\mathrm{C}_{6} \mathrm{H}_{15} \mathrm{O}_{6}$ & 1.2 & sat'd, $\left[G_{182}+\mathrm{H}\right]^{+} \mathrm{CAD}:-\mathrm{H}_{2} \mathrm{O},-2 \mathrm{H}_{2} \mathrm{O},-3 \mathrm{H}_{2} \mathrm{O}$ \\
\hline 185.10251 & $\mathrm{C}_{6} \mathrm{H}_{17} \mathrm{O}_{6}$ & 65 & Gly aggregate: $[2 \mathrm{Gly}+\mathrm{H}]^{+}$, fragment of $\left[\mathrm{G}_{122}+\mathrm{Gly}+\mathrm{H}\right]^{+} \mathrm{CAD}$ : [Gly+H$]^{+},\left[\mathrm{Gly}-\mathrm{H}_{2} \mathrm{O}+\mathrm{H}\right]^{+}$ \\
\hline 187.10676 & $\mathrm{C}_{6} \mathrm{H}_{12} \mathrm{O}_{5}{ }^{18} \mathrm{O}$ & 0.7 & {$[2 \mathrm{Gly}+\mathrm{H}]^{+}$} \\
\hline 187.06065 & $\mathrm{C}_{4} \mathrm{H}_{4} \mathrm{O}_{5}$ & 0.1 & \\
\hline 189.07630 & $\mathrm{C}_{8} \mathrm{H}_{43} \mathrm{O}_{5}$ & 0.05 & Gly aggregate: $\left[\left\{G_{182}-3 \mathrm{H}_{2} \mathrm{O}-\mathrm{CH}_{3} \mathrm{OH}\right\}+\mathrm{Gly}+\mathrm{H}\right]^{+}$ \\
\hline 191.09195 & $\mathrm{C}_{8} \mathrm{H}_{15} \mathrm{O}_{5}$ & 0.07 & $\begin{array}{l}\text { Gly aggregate: }\left[\left\{G_{152}-3 \mathrm{H}_{2} \mathrm{O}++\mathrm{Gly}+\mathrm{H}\right]^{+} \text {, fragment of }\left[\left\{G_{152}-2 \mathrm{H}_{2} \mathrm{O}\right\}+\mathrm{Gly}+\mathrm{H}\right]^{+} \text {metastable: }\right. \\
-\mathrm{H}_{2} \mathrm{OCAD}:-\mathrm{H}_{2} \mathrm{O},-2 \mathrm{H}_{2} \mathrm{O},-\mathrm{Gly}\end{array}$ \\
\hline 193.10760 & $\mathrm{C}_{\mathrm{R}} \mathrm{H}_{17} \mathrm{O}_{5}$ & 0.03 & Gly aggregate: $\left[\left\{G_{136}-2 \mathrm{H}_{2} \mathrm{O}\right\}+\mathrm{Gly}+\mathrm{H}\right]^{+}$metastable: $-\mathrm{H}_{2} \mathrm{O},-\mathrm{Gly}$ \\
\hline 195.08686 & $\mathrm{C}_{7} \mathrm{H}_{15} \mathrm{O}_{6}$ & 0.08 & Gly aggregate: $\left[\left\{G_{152}-\mathrm{H}_{2} \mathrm{O}-\mathrm{CH}_{3} \mathrm{OH}\right\}+\mathrm{Gly}+\mathrm{H}\right]^{+}$ \\
\hline 197.10251 & $\mathrm{C}_{7} \mathrm{H}_{1}, \mathrm{O}_{6}$ & 0.3 & sat'd, Gly aggregate: $\left[\left[\mathrm{G}_{12 x}-\mathrm{H}_{2} \mathrm{O}\right\}+\mathrm{Gly}+\mathrm{H}^{+}\right.$metastable: $-\mathrm{H}_{2} \mathrm{O},[\mathrm{Gly}+\mathrm{H}]^{+},-2 \mathrm{H}_{2} \mathrm{O},-\mathrm{Gly}$ \\
\hline 199.11816 & $\mathrm{C}_{7} \mathrm{H}_{19} \mathrm{O}_{6}$ & 0.4 & $\begin{array}{l}\text { sat'd, Gly aggregate: }\left[\left(G_{100}+\mathrm{H}\right)+\mathrm{Gly}\right]^{+} \mathrm{CAD}:\left[G_{106}+\mathrm{H}\right]^{+} \\
{\left[G_{100}-\mathrm{H}_{2} \mathrm{O}+\mathrm{H}\right]^{+},\left[\mathrm{Gly}+[\mathrm{H}]^{+}\right.}\end{array}$ \\
\hline 201.07630 & $\mathrm{C}_{9} \mathrm{H}_{13} \mathrm{O}_{5}$ & 0.07 & Gly aggregate \\
\hline 203.09195 & $\mathrm{C}_{9} \mathrm{H}_{15} \mathrm{O}_{5}$ & 0.1 & $\begin{array}{l}\text { Gly aggregate: }\left[\left\{G_{18 z}-4 \mathrm{H}_{2} \mathrm{O}\right\}+\mathrm{Gly}+\mathrm{H}\right]^{+} \text {, fragment of } \\
{\left[\left\{G_{18 z}-3 \mathrm{H}_{2} \mathrm{O}\right\}+\mathrm{Gly}+\mathrm{H}\right]^{+}}\end{array}$ \\
\hline 205.10760 & $\mathrm{C}_{5} \mathrm{H}_{19} \mathrm{O}_{5}$ & 0.03 & Gly aggregate: $\left[\left[\mathrm{T}_{166}-3 \mathrm{H}_{2} \mathrm{O}\right]+\mathrm{Gly}+\mathrm{H}\right]^{+}$, fragment of $\left[\left\{\mathrm{G}_{166}-2 \mathrm{H}_{2} \mathrm{O}\right\}+\mathrm{Gly}+\mathrm{H}\right]^{+}$ \\
\hline 207.08686 & $\mathrm{C}_{\mathrm{B}} \mathrm{H}_{15} \mathrm{O}_{6}$ & 0.07 & Gly aggregate: $\left[\left[\mathrm{G}_{182}-2 \mathrm{H}_{2} \mathrm{O}-\mathrm{CH}_{3} \mathrm{OH}\right]+\mathrm{Gly}+\mathrm{H}\right]^{+}$ \\
\hline 209.10251 & $\mathrm{C}_{8} \mathrm{H}_{17} \mathrm{O}_{6}$ & 0.08 & $\begin{array}{l}\text { Gly aggregate: }\left[\left[\mathrm{G}_{152}-2 \mathrm{H}_{2} \mathrm{O}\right\}+\mathrm{Gly}+\mathrm{H}\right]^{+} \text {, fragment of }\left[\left\{\mathrm{G}_{153}-\mathrm{H}_{2} \mathrm{O}\right\}+\mathrm{Gly}+\mathrm{H}\right]^{+} \text {metastable: }-\mathrm{H}_{2} \mathrm{O} \text {. } \\
-2 \mathrm{H}_{2} \mathrm{O} \text {, - Gly CAD: Gly, }-\mathrm{H}_{2} \mathrm{O},-2 \mathrm{H}_{2} \mathrm{O} 80 \% \mathrm{CAD}: \mathrm{CAD}:-\mathrm{Gly},-2 \mathrm{H}_{2} \mathrm{O},-\mathrm{H}_{2} \mathrm{O}\end{array}$ \\
\hline 211.11816 & $\mathrm{C}_{8} \mathrm{H}_{19} \mathrm{O}_{6}$ & 0.1 & sal'd, Gly aggregate: $\left[\left\{G_{136}-\mathrm{H}_{2} \mathrm{O}+\mathrm{Gly}+\mathrm{H}\right]^{+} \mathrm{CAD}:-\mathrm{H}_{2} \mathrm{O},-\mathrm{Gly},\left[G_{136}-2 \mathrm{H}_{2} \mathrm{O}+\mathrm{H}\right]^{+}\right.$ \\
\hline 215.11308 & $\mathrm{C}_{7} \mathrm{H}_{19} \mathrm{O}_{7}$ & 0.7 & $\begin{array}{l}\text { sat'd, Gly aggregate: }\left[G_{122}+\mathrm{Gly}+\mathrm{H}\right]^{+} \mathrm{CAD}:\left[G_{122}+\mathrm{H}\right]^{+},-\mathrm{H}_{2} \mathrm{CO},\left[G_{122}-\mathrm{H}_{2} \mathrm{O}+\mathrm{H}\right]^{+} .[\mathrm{Gly}+\mathrm{H}]^{+} \text {, no } \\
\text { detectable } \mathrm{H}_{2} \mathrm{O} \text { loss }\end{array}$ \\
\hline 221.10251 & $\mathrm{C}_{9} \mathrm{H}_{17} \mathrm{O}_{6}$ & 0.07 & $\begin{array}{l}\text { Gly aggregate: }\left[\left\{G_{18 z}-3 \mathrm{H}_{2} \mathrm{O}\right\}+\mathrm{Gly}+\mathrm{H}\right]^{+} \mathrm{CAD}:-\mathrm{H}_{2} \mathrm{O},\left[G_{182}-3 \mathrm{H}_{2} \mathrm{O}+\mathrm{H}\right]^{+},-\left(\mathrm{H}_{2} \mathrm{O}+\mathrm{H}_{2} \mathrm{CO}\right) \\
-2 \mathrm{H}_{2} \mathrm{O} 80 \% \mathrm{CAD}:-\mathrm{H}_{2} \mathrm{O},\left[G_{\mathrm{LEz}}-3 \mathrm{H}_{2} \mathrm{O}+\mathrm{H}\right]^{+},-2 \mathrm{H}_{2} \mathrm{O},-\left(\mathrm{H}_{2} \mathrm{O}+\mathrm{H}_{2} \mathrm{CO}\right)\end{array}$ \\
\hline 223.11816 & $\mathrm{C}_{9} \mathrm{H}_{19} \mathrm{O}_{6}$ & 0.1 & $\begin{array}{l}\text { Gly aggregate: }\left[\left\{G_{166}-2 \mathrm{H}_{2} \mathrm{O}\right\}+\mathrm{Oly}+\mathrm{H}\right]^{+} \text {, fragnemt of }\left[\left[G_{166}-1 \mathrm{I}_{2} \mathrm{O}\right\}+\mathrm{Gly}+\mathrm{III}\right]^{+} \mathrm{CAD}:-\mathrm{Gly} \text {, } \\
-\mathrm{H}_{2} \mathrm{O}\end{array}$ \\
\hline 227.11308 & $\mathrm{C}_{\mathrm{8}} \mathrm{H}_{19} \mathrm{O}_{7}$ & 0.1 & $\begin{array}{l}\text { Gly aggregate: }\left[\left\{G_{152}-\mathrm{H}_{2} \mathrm{O}\right\}+\mathrm{Gly}+\mathrm{H}\right]^{+} \text {metastable: }-\mathrm{H}_{2} \mathrm{O},-\mathrm{Gly} 8 \mathrm{O} \% \mathrm{CAD}:-\mathrm{H}_{2} \mathrm{O},-\mathrm{Gly} \text {, } \\
-\left(\mathrm{Gly}+\mathrm{H}_{2} \mathrm{O}\right)\end{array}$ \\
\hline 229.12873 & $\mathrm{C}_{9} \mathrm{H}_{21} \mathrm{O}$, & 0.2 & Gly aggregate: $\left[\left(G_{136}+\mathrm{H}\right)+\mathrm{Gly}\right]^{+}$ \\
\hline
\end{tabular}


Table 1-(continued)

\begin{tabular}{|c|c|c|c|}
\hline$m / z$ & Formula & Abundance & Comments and lon Origin \\
\hline 237.13381 & $\mathrm{C}_{10} \mathrm{H}_{21} \mathrm{O}_{6}$ & 0.03 & Gly aggregate: $\left[\left\{G_{106}-3 \mathrm{H}_{2} \mathrm{O}\right\}+2 \mathrm{Gly}+\mathrm{H}\right]^{+}$metastable $-\mathrm{H}_{2} \mathrm{O},-$ Gly CAD: $-\mathrm{Gly},-\mathrm{H}_{2} \mathrm{O}$ \\
\hline 239.11308 & $\mathrm{C}_{9} \mathrm{H}_{19} \mathrm{O}_{7}$ & 0.1 & $\left.\left[\mid G_{1 a_{2}}-2 \mathrm{H}_{2} \mathrm{O}\right\}+\mathrm{Gly}+\mathrm{H}\right]^{+}$metastable: $-\mathrm{H}_{2} \mathrm{O},-\mathrm{Gly},-2 \mathrm{H}_{2} \mathrm{O}$ \\
\hline 241.12873 & $\mathrm{C}_{\mathbf{g}} \mathrm{H}_{21} \mathrm{O}_{7}$ & 0.4 & Gly aggregate: $\left[\left\{G_{166}-\mathrm{H}_{2} \mathrm{O}\right\}+\mathrm{Gly}+\mathrm{H}\right]^{+} \mathrm{CAD}:-\mathrm{H}_{2} \mathrm{O},-\mathrm{Gly}$ \\
\hline 245.12364 & $\mathrm{C}_{8} \mathrm{H}_{21} \mathrm{O}_{8}$ & 0.6 & $\begin{array}{l}\text { Gly aggregate: }\left[\left(G_{153}+\mathrm{H}\right)+\mathrm{Gly}\right]^{+} \text {and }\left[G_{60}+2 \mathrm{Gly}+\mathrm{H}\right]^{+} \text {metastable: }-\mathrm{Gly},-G_{60} \mathrm{CAD}:-\mathrm{Gly},-G_{403} \\
{\left[\mathrm{Gly}+\mathrm{H}^{+},-\mathrm{H}_{2} \mathrm{O} \text { (low abundance), and }-\mathrm{H}_{2} \mathrm{CO} \text { (low abundance) }\right.}\end{array}$ \\
\hline 253.12873 & $\mathrm{C}_{10} \mathrm{H}_{2 \mathrm{t}} \mathrm{O}_{7}$ & 0.03 & Gly aggregate: $\left[\left(G_{12 z}-3 \mathrm{H}_{2} \mathrm{O}\right\}+2 \mathrm{Gly}+\mathrm{H}\right]^{+} \mathrm{CAD}:-\mathrm{Gly},-\mathrm{H}_{2} \mathrm{O},-\left(\mathrm{H}_{2} \mathrm{O}+\mathrm{Gly}\right),-2 \mathrm{Gly}$ \\
\hline 257.12364 & $\mathrm{C}_{9} \mathrm{H}_{21} \mathrm{O}_{8}$ & 0.2 & $\begin{array}{l}\text { Gly aggregate: }\left[\left\{G_{182}-\mathrm{H}_{2} \mathrm{O}\right\}+\mathrm{Gly}+\mathrm{H}\right]^{+} \text {and }\left[\left\{G_{90}-\mathrm{H}_{2} \mathrm{O}\right\}+2 \mathrm{Gly}+\mathrm{H}\right]^{+} \text {metastable: }-\mathrm{H}_{2} \mathrm{O},-\mathrm{Gly} \text {, } \\
-\left(G_{90}-\mathrm{H}_{2} \mathrm{O}\right)\end{array}$ \\
\hline 259.13929 & $\mathrm{C}_{9} \mathrm{H}_{23} \mathrm{O}_{3}$ & 0.4 & $\begin{array}{l}\text { sat'd, TD, Gly aggregate: }\left[\left(G_{168}+H\right)+G l y\right]^{+} \text {and }\left[G_{74}+2 G l y+H\right]^{+} \mathrm{CAD}:-\mathrm{Gly},-G_{74}-\mathrm{H}_{x} \mathrm{O} \text { (low } \\
\text { abundance), }-\mathrm{H}_{2} \mathrm{CO} \text { (low abundance) }\end{array}$ \\
\hline 265.12873 & $\mathrm{C}_{11} \mathrm{H}_{21} \mathrm{O}_{7}$ & 0.06 & Gly aggregate: $\left[\left\{G_{152}-4 \mathrm{H}_{2} \mathrm{O}\right\}+2 \mathrm{Gly}+\mathrm{H}\right]^{+}$fragment of $\left[\left\{G_{152}-3 \mathrm{H}_{2} \mathrm{O}\right\}+2 \mathrm{Gly}+\mathrm{H}\right]^{+}$ \\
\hline 275.13421 & $\mathrm{C}_{9} \mathrm{H}_{\mathbf{2 3}} \mathrm{O}$ & 1.0 & $\begin{array}{l}{\left[G_{192}+G_{122}+\mathrm{H}\right]^{+} \text {and Gly aggregate: }\left[\left(G_{182}+\mathrm{H}\right)+\mathrm{Gly}\right]^{+} \text {and }\left[G_{90}+2 \mathrm{Gly}+\mathrm{H}\right]^{+} \mathrm{CAD}:-\mathrm{Gly},-G_{90}} \\
{\left[G_{182}-\mathrm{H}_{2} \mathrm{O}+\mathrm{H}\right]^{+},\left[G_{122}+\mathrm{H}\right]^{+},\left[G_{192}+\mathrm{H}\right]^{+},[\mathrm{Gly}+\mathrm{H}]^{+}}\end{array}$ \\
\hline 277.14986 & $\mathrm{C}_{9} \mathrm{H}_{23} \mathrm{O}_{9}$ & 6.3 & Gly aggregate: $[3 \mathrm{Gly}+\mathrm{H}]^{+}$, fragment of $\left[\left(G_{122}+\mathrm{H}\right)+2 \mathrm{Glyl}^{+}\right.$ \\
\hline 283.13929 & $\mathrm{C}_{11} \mathrm{H}_{23} \mathrm{O}_{8}$ & 0.08 & $\begin{array}{l}\text { Gly aggregate: }\left[\left\{G_{152}-3 \mathrm{H}_{2} \mathrm{O}\right\}+2 \mathrm{Gly}+\mathrm{H}\right]^{+} \text {metastable: }-\mathrm{H}_{2} \mathrm{O},-\mathrm{Gly} \text { CAD: }-\mathrm{Gly},-\mathrm{H}_{2} \mathrm{O} 80 \% \\
\text { CAD: }-\mathrm{Gly},-\mathrm{H}_{2} \mathrm{O},-\left(\mathrm{H}_{2} \mathrm{O}+\mathrm{Gly}\right),\left[\mathrm{G}_{152}-3 \mathrm{H}_{2} \mathrm{O}+\mathrm{H}\right]^{+} \text {(low abundance) }\end{array}$ \\
\hline 289.14986 & $\mathrm{C}_{10} \mathrm{H}_{23} \mathrm{O}_{9}$ & 0.2 & sat'd, Gly aggregate: $\left[\left\{G_{122}-\mathrm{H}_{2} \mathrm{O}\right\}+2 \mathrm{Gly}+\mathrm{H}\right]^{+}$ \\
\hline 291.16551 & $\mathrm{C}_{10} \mathrm{H}_{27} \mathrm{O}_{9}$ & 0.1 & sat'd, Gly aggregate: $\left[\left(G_{106}+\mathrm{H}\right)+2 \mathrm{Gly}\right]^{+}$ \\
\hline 297.15494 & $\mathrm{C}_{12} \mathrm{H}_{23} \mathrm{O}_{8}$ & 0.04 & $\begin{array}{l}\text { Gly aggregate: }\left[\left\{G_{166}-3 \mathrm{H}_{2} \mathrm{O}\right\}+2 \mathrm{Gly}+\mathrm{H}\right]^{+}, \text {fragment of }\left[\left\{G_{166}-2 \mathrm{H}_{2} \mathrm{O}\right\}+2 \mathrm{Gly}+\mathrm{H}\right]^{+} \\
\text {CAD: }-\mathrm{Gly},-\mathrm{H}_{2} \mathrm{O},-\left(\mathrm{H}_{2} \mathrm{O}+\mathrm{Gly}\right),\left[G_{166}-3 \mathrm{H}_{2} \mathrm{O}+\mathrm{H}\right]^{+}\end{array}$ \\
\hline 301.14986 & $\mathrm{C}_{11} \mathrm{H}_{25} \mathrm{O}_{9}$ & 0.1 & $\begin{array}{l}\text { Gly aggregate: }\left[\left\{G_{152}-2 \mathrm{H}_{2} \mathrm{O}\right\}+2 \mathrm{Gly}+\mathrm{H}^{+} \text {metastable: }-\mathrm{H}_{2} \mathrm{O},-\mathrm{Gly},-\left(\mathrm{H}_{2} \mathrm{O}+\mathrm{Gly}\right) 80 \% \mathrm{CAD}\right. \\
-\mathrm{H}_{2} \mathrm{O},-\mathrm{Gly},-\left(\mathrm{Gly}_{2}+\mathrm{H}_{2} \mathrm{O}\right),-\left(\mathrm{Gly}+2 \mathrm{H}_{2} \mathrm{O}\right),\left[G_{152}-2 \mathrm{H}_{2} \mathrm{O}+\mathrm{H}\right]^{+}\end{array}$ \\
\hline 307.16042 & $\mathrm{C}_{10} \mathrm{H}_{27} \mathrm{O}_{10}$ & 0.3 & Gly aggregate: $\left[\left(G_{122}+\mathrm{H}\right)+2 \mathrm{G}\right]^{+} \mathrm{CAD}:-\mathrm{Gly},-\left(\mathrm{Gly}+\mathrm{H}_{2} \mathrm{CO}\right),-2 \mathrm{Gly},[\mathrm{Gly}+\mathrm{H}]^{+}$ \\
\hline 313.14986 & $\mathrm{C}_{12} \mathrm{H}_{25} \mathrm{O}_{9}$ & 0.08 & $\begin{array}{l}\text { Gly aggregate: }\left[\left\{\mathrm{G}_{1 \mathrm{tz}}-3 \mathrm{H}_{2} \mathrm{O}\right\}+2 \mathrm{Gly}+\mathrm{H}\right]^{+} \mathrm{CAD}:-\mathrm{Gly},-\mathrm{H}_{2} \mathrm{O},-\left(\mathrm{H}_{2} \mathrm{O}+\mathrm{Gly}\right),-2 \mathrm{Gly} 80 \% \mathrm{CAD} \\
-\mathrm{Gly},-\mathrm{H}_{2} \mathrm{O},-\left(\mathrm{H}_{2} \mathrm{O}+\mathrm{Gly}\right),-2 \mathrm{Gly}\end{array}$ \\
\hline 315.16551 & $\mathrm{C}_{12} \mathrm{H}_{21} \mathrm{O}$ & 0.1 & Gly aggregate: $\left[\left\{G_{166}-2 \mathrm{H}_{2} \mathrm{O}\right\}+2 \mathrm{Gly}+\mathrm{H}\right]^{+} 80 \% \mathrm{CAD}:-\mathrm{Gly},\left[G_{166}-2 \mathrm{H}_{2} \mathrm{O}+\mathrm{H}\right]^{+},-\mathrm{H}_{2} \mathrm{O}$ \\
\hline 319.16042 & $\mathrm{C}_{11} \mathrm{H}_{27} \mathrm{O}_{10}$ & 0.06 & $\begin{array}{l}\text { Gly aggregate: }\left[\left\{G_{152}-\mathrm{H}_{2} \mathrm{O}\right\}+2 \mathrm{Gly}+\mathrm{H}\right]^{+} \text {metastable: }-\mathrm{H}_{2} \mathrm{O},-\mathrm{Gly} 80 \% \mathrm{CAD}:-\mathrm{Gly} \text {, } \\
-\left(\mathrm{H}_{2} \mathrm{O}+\mathrm{Gly}\right) \ldots\left[\mathrm{G}_{152}-\mathrm{H}_{2} \mathrm{O}+\mathrm{H}\right]^{+}\end{array}$ \\
\hline 321.17607 & $\mathrm{C}_{11} \mathrm{H}_{29} \mathrm{O}_{10}$ & 0.08 & Gly aggregate: $\left[\left(G_{136}+H\right)+2 G l y\right]^{+}$ \\
\hline 333.17607 & $\mathrm{C}_{12} \mathrm{H}_{29} \mathrm{O}_{10}$ & 0.2 & Gly aggregate: $\left[\left\{G_{166}-\mathrm{H}_{2} \mathrm{O}\right\}+2 \mathrm{Gly}+\mathrm{H}\right]^{+} \mathrm{CAD}:-\mathrm{Gly},-\mathrm{H}_{2} \mathrm{O},-\left(\mathrm{H}_{2} \mathrm{O}+\mathrm{Gly}\right),\left[G_{166}-\mathrm{H}_{2} \mathrm{O}+\mathrm{H}\right]^{+}$ \\
\hline 337.17099 & $\mathrm{C}_{11} \mathrm{H}_{29} \mathrm{O}_{11}$ & 0.2 & $\begin{array}{l}\text { Gly aggregate: }\left[\left(G_{153}+\mathrm{H}\right)+2 \mathrm{Gly}\right]^{+} \text {and }\left[G_{60}+2 \mathrm{Gly}+\mathrm{H}\right]^{+} \text {metustable: }-\mathrm{H}_{2} \mathrm{O},-\mathrm{Gly}_{+}-G_{60} 80 \% \mathrm{CAD}: \\
-\mathrm{Gly},-2 \mathrm{Gly},-\left(G_{60}+\mathrm{Gly}\right),[\mathrm{Gly}+\mathrm{H}]^{+} \ldots-G_{60}\end{array}$ \\
\hline 349.17099 & $\mathrm{C}_{12} \mathrm{H}_{29} \mathrm{O}_{11}$ & 0.2 & $\begin{array}{l}\text { Gly aggregate: }\left[\left\{G_{182}-\mathrm{H}_{2} \mathrm{O}\right\}+2 \mathrm{Gly}+\mathrm{H}\right]^{+} \text {and }\left[\left\{G_{90}-\mathrm{H}_{2} \mathrm{O}\right\}+3 \mathrm{Gly}+\mathrm{H}\right]^{+} \text {metastable: }-\mathrm{Gly},-\mathrm{H}_{2} \mathrm{O} \text {, } \\
-\left(G_{90}-\mathrm{H}_{2} \mathrm{O}\right)\end{array}$ \\
\hline 351.18664 & $\mathrm{C}_{12} \mathrm{H}_{31} \mathrm{O}_{11}$ & 0.2 & sat'd, Gly aggregate: $\left[\left(G_{166}+\mathrm{H}\right)+2 \mathrm{Gly}\right]^{+}$and $\left[G_{74}+3 \mathrm{Gly}+\mathrm{H}\right]^{+}$ \\
\hline 367.18155 & $\mathrm{C}_{12} \mathrm{H}_{31} \mathrm{O}_{12}$ & 0.4 & $\begin{array}{l}\text { Gly aggregate: }\left[\left(G_{162}+\mathrm{H}\right)+\mathrm{Gly}\right]^{+},\left[G_{90}+3 \mathrm{Gly}+\mathrm{H}\right]^{+} \text {and }\left[G_{152}+G_{\mathrm{tz2}}+\mathrm{Gly}+\mathrm{H}\right]^{+} \mathrm{CAD}:-\mathrm{Gly},-2 \mathrm{Gly} \text {, } \\
-\left(\mathrm{Gly}+G_{90}\right),-G_{90,}[\mathrm{Gly}+\mathrm{H}]^{+},\left[G_{132}-\mathrm{H}_{2} \mathrm{O}+\mathrm{H}\right]^{+},\left[G_{152}+\mathrm{H}\right]^{+}\end{array}$ \\
\hline 369.19720 & $\mathrm{C}_{12} \mathrm{H}_{33} \mathrm{O}_{12}$ & 1.2 & Gly aggregate: $[4 \mathrm{Gly}+\mathrm{H}]^{+}$ \\
\hline 441.21833 & $\mathrm{C}_{15} \mathrm{H}_{37} \mathrm{O}_{14}$ & 0.09 & Gly aggregate: $\left[\left\{G_{182}-\mathrm{H}_{2} \mathrm{O}\right\}+3 \mathrm{Gly}+\mathrm{H}\right]^{+}$and $\left[\left\{G_{90}-\mathrm{H}_{2} \mathrm{O}\right\}+4 \mathrm{Gly}+\mathrm{H}\right]^{+}$metastable: $-\mathrm{Gly},-\mathrm{H}_{2} \mathrm{O}$ \\
\hline 491.25511 & $\mathrm{C}_{16} \mathrm{H}_{43} \mathrm{O}_{16}$ & 0.07 & Gly aggregate: $\left[\left(G_{122}+\mathrm{H}\right)+3 \mathrm{Gly}\right]^{+}$metastable: $-\mathrm{Gly},-\mathrm{H}_{2} \mathrm{O}$ \\
\hline
\end{tabular}

\section{Theory for Formation of Background Ions}

Field [24] proposed that C-centered free radicals are produced from glycerol during the bombardment event. These radicals couple to produce new molecular species, which were determined off-line following bombardment. Extending the investigation by Field, Keough et al. [25] used capillary gas chromatography/ mass spectrometry to determine the bombardment products and compared them to those produced from $\gamma$ irradiation. The results from both of these investigations support the hypothesis that matrix-derived free radicals are produced during the bombardment event, and that these radicals couple to produce new, covalently bonded molecules.

To aid in the discussion of the results of our study, the following notation is used. Species produced as a result of particle bombardment are denoted by an italicized letter, coming from the first letter of the name of the matrix, with the formula weight given by a subscript; radical species are distinguished by a dot. The notation is not specific for structure, only for molecular weight. Isomeric matrix-derived radicals are formed and couple to produce structural isomers [25]. 
Table 2. Tabulated spectrum of glycerol negative background ions

\begin{tabular}{|c|c|c|c|}
\hline $\mathrm{m} / \mathrm{z}$ & Formula & Abundance & Comments and lon Origin \\
\hline 12.00000 & C & 0.2 & $-\bullet$ \\
\hline 13.00782 & $\mathrm{CH}$ & 1.1 & \\
\hline 14.01565 & $\mathrm{CH}_{2}$ & 0.3 & - \\
\hline 15.99491 & 0 & 6.0 & $-\infty$ \\
\hline 17.00274 & OH & 8.5 & $\mathrm{TD}$ \\
\hline 24.00000 & $\mathrm{C}_{2}$ & 0.7 & $\cdots$ \\
\hline 25.00782 & $\mathrm{C}_{2} \mathrm{H}$ & 2.8 & \\
\hline 27.02347 & $\mathrm{C}_{2} \mathrm{H}_{3}$ & 0.1 & \\
\hline 29.00274 & CHO & 0.2 & \\
\hline 31.01839 & $\mathrm{CH}_{3} \mathrm{O}$ & 0.4 & TD \\
\hline 31.98983 & $\mathrm{O}_{2}$ & 0.2 & $\cdots$ \\
\hline 32.99765 & $\mathrm{HO}_{2}$ & 0.2 & \\
\hline 37.00782 & $\mathrm{C}_{3} \mathrm{H}$ & 0.2 & \\
\hline 39.99491 & $\mathrm{C}_{2} \mathrm{O}$ & 0.4 & $-\bullet$ \\
\hline 41.00274 & $\mathrm{C}_{2} \mathrm{HO}$ & 2.7 & \\
\hline 43.01839 & $\mathrm{C}_{2} \mathrm{H}_{3} \mathrm{O}$ & 2.0 & TD \\
\hline 44.99765 & $\mathrm{CHO}_{3}$ & 1.2 & \\
\hline 53.00274 & $\mathrm{C}_{3} \mathrm{HO}$ & 0.3 & \\
\hline 55.05477 & $\mathrm{C}_{3} \mathrm{H}_{3} \mathrm{O}$ & 0.8 & $\mathrm{TD}$ \\
\hline 56.99765 & $\mathrm{C}_{2} \mathrm{HO}_{2}$ & 0.5 & \\
\hline 56.99765 & $\mathrm{C}_{2} \mathrm{HO}_{2}$ & 0.1 & \\
\hline 57.03404 & $\mathrm{C}_{3} \mathrm{H}_{5} \mathrm{O}$ & 0.05 & \\
\hline 58.00548 & $\mathrm{C}_{2} \mathrm{H}_{2} \mathrm{O}_{2}$ & 2.3 & $\cdots$ \\
\hline 59.01330 & $\mathrm{C}_{2} \mathrm{H}_{3} \mathrm{O}_{2}$ & 18.4 & \\
\hline 68.99765 & $\mathrm{C}_{3} \mathrm{HO}_{2}$ & 0.3 & \\
\hline 69.03404 & $\mathrm{C}_{4} \mathrm{H}_{5} \mathrm{O}$ & 0.1 & \\
\hline 70.00547 & $\mathrm{C}_{3} \mathrm{H}_{2} \mathrm{O}_{2}$ & 0.3 & $\rightarrow$ \\
\hline 71.01330 & $\mathrm{C}_{3} \mathrm{H}_{3} \mathrm{O}_{2}$ & 8.3 & {$\left[G_{90}-\mathrm{H}_{2} \mathrm{O}-\mathrm{H}^{-}\right.$} \\
\hline 72.02112 & $\mathrm{C}_{3} \mathrm{H}_{4} \mathrm{O}_{2}$ & 1.0 & $\cdots,\left[\mathrm{Gly}-\mathrm{H}-\mathrm{H}_{2} \mathrm{O}-\mathrm{H}\right]^{-}$ \\
\hline 73.02895 & $\mathrm{C}_{3} \mathrm{H}_{3} \mathrm{O}_{2}$ & 2.6 & $\mathrm{TD},\left[\mathrm{Gly}-\mathrm{H}_{2} \mathrm{O}-\mathrm{H}\right]^{-}$ \\
\hline 75.00822 & $\mathrm{C}_{2} \mathrm{H}_{3} \mathrm{O}_{3}$ & 0.5 & \\
\hline 83.01330 & $\mathrm{C}_{4} \mathrm{H}_{3} \mathrm{O}_{2}$ & 0.2 & abundance increases with time in beam \\
\hline 84.02112 & $\mathrm{C}_{4} \mathrm{H}_{4} \mathrm{O}_{2}$ & 0.1 & $-\bullet$ \\
\hline 85.02895 & $\mathrm{C}_{4} \mathrm{H}_{9} \mathrm{O}_{2}$ & 0.7 & \\
\hline 86.00039 & $\mathrm{C}_{3} \mathrm{H}_{2} \mathrm{O}_{3}$ & 0.3 & $\because$ \\
\hline 87.00822 & $\mathrm{C}_{3} \mathrm{H}_{3} \mathrm{O}_{3}$ & 1.9 & \\
\hline 88.01604 & $\mathrm{C}_{3} \mathrm{H}_{4} \mathrm{O}_{3}$ & 3.1 & $-\bullet$ \\
\hline 89.02387 & $\mathrm{C}_{3} \mathrm{H}_{5} \mathrm{O}_{3}$ & 16.1 & {$\left[G_{90}-\mathrm{H}^{-}\right.$, metastable: $-\mathrm{H}_{2} \mathrm{O},-\mathrm{H}_{2} \mathrm{CO},-\mathrm{H}$} \\
\hline 91.03952 & $\mathrm{C}_{3} \mathrm{H}_{7} \mathrm{O}_{3}$ & 77.7 & sat'd, IGly $-\mathrm{HI}^{-}$metastable: $-\mathrm{H}_{2}-\mathrm{CH}_{3} \mathrm{OH} \mathrm{CAD}:-\mathrm{H}_{2},-\mathrm{CH}_{3} \mathrm{OH}$ or $-\left(\mathrm{H}_{2}+\mathrm{H}_{2} \mathrm{CO}\right),-\left(\mathrm{H}_{2}+\mathrm{H}_{2} \mathrm{O}\right)$ \\
\hline 93.04377 & $\mathrm{C}_{3} \mathrm{H}_{7} \mathrm{O}_{2}{ }^{18} \mathrm{O}$ & 0.3 & {$[\mathrm{Gly}-\mathrm{H}]^{-}$} \\
\hline 97.02895 & $\mathrm{C}_{3} \mathrm{H}_{3} \mathrm{O}_{2}$ & 0.3 & \\
\hline 98.03677 & $\mathrm{C}_{5} \mathrm{H}_{6} \mathrm{O}_{2}$ & 0.09 & $-\bullet$ \\
\hline 99.00822 & $\mathrm{C}_{4} \mathrm{H}_{3} \mathrm{O}_{3}$ & 0.2 & abundance increases with time in beam \\
\hline 99.04460 & $\mathrm{C}_{5} \mathrm{H}_{7} \mathrm{O}_{2}$ & 0.1 & abundance increases with time in beam \\
\hline
\end{tabular}

For aggregate species, braces are used as a guide to indicate the formal composition of the ion, but isomeric structures may also contribute to the ion population. lon urigins are based on tandern mass spectra and teluental compositions. See text for further discussion. -- = racical anion

TD = this ion that may also arise from deprotonation of a thermal decomposition product.

sat ${ }^{\prime}=$ a saturated species or an anion neutral complex composed of an unsaturated molecule and one or more glycerol molecules.

metastable or CAD - the losces, product ions, or the mase-to-charge ratio value of the product ions that result from metastable ion or CA decompositions. The ions are listed by decreasing abundance. 
Table 2-(continued)

\begin{tabular}{|c|c|c|c|}
\hline$m / 2$ & Formula & Abundance & Comments and lon Origin \\
\hline 100.01604 & $\mathrm{C}_{4} \mathrm{H}_{4} \mathrm{O}_{3}$ & 0.1 & $-\bullet$ \\
\hline 101.02387 & $\mathrm{C}_{4} \mathrm{H}_{5} \mathrm{O}_{3}$ & 0.6 & $\mathrm{CAD}:-\mathrm{H}_{2} \mathrm{O},-\mathrm{CO}$ \\
\hline 103.03952 & $\mathrm{C}_{4} \mathrm{H}_{7} \mathrm{O}_{3}$ & 0.3 & \\
\hline 103.00313 & $\mathrm{C}_{3} \mathrm{H}_{3} \mathrm{O}_{4}$ & 0.08 & \\
\hline 105.01878 & $\mathrm{C}_{3} \mathrm{H}_{9} \mathrm{O}_{4}$ & 0.5 & \\
\hline 105.05517 & $\mathrm{C}_{4} \mathrm{H}_{9} \mathrm{O}_{3}$ & 0.1 & sat'd, CAD: $-\mathrm{H}_{2} \mathrm{CO},-\mathrm{H}_{2} \mathrm{O}, 59$ \\
\hline 107.03443 & $\mathrm{C}_{3} \mathrm{H}_{9} \mathrm{O}_{4}$ & 0.1 & sat'd \\
\hline 109.05008 & $\mathrm{C}_{3} \mathrm{H}_{9} \mathrm{O}_{4}$ & 0.9 & sat'd \\
\hline 111.04460 & $\mathrm{C}_{6} \mathrm{H}_{7} \mathrm{O}_{2}$ & 0.1 & \\
\hline 111.00822 & $\mathrm{C}_{5} \mathrm{H}_{3} \mathrm{O}_{3}$ & 0.07 & abundance increases with time in beam \\
\hline 113.02387 & $\mathrm{C}_{5} \mathrm{H}_{5} \mathrm{O}_{3}$ & 0.4 & \\
\hline 115.03952 & $\mathrm{C}_{5} \mathrm{H}_{7} \mathrm{O}_{3}$ & 0.4 & abundance increases with time in bearn \\
\hline 115.00313 & $\mathrm{C}_{4} \mathrm{H}_{3} \mathrm{O}_{4}$ & 0.1 & \\
\hline 117.05517 & $\mathrm{C}_{3} \mathrm{H}_{9} \mathrm{O}_{3}$ & 0.1 & \\
\hline 117.01878 & $\mathrm{C}_{4} \mathrm{H}_{5} \mathrm{O}_{4}$ & 0.05 & \\
\hline 118.02660 & $\mathrm{C}_{4} \mathrm{H}_{6} \mathrm{O}_{4}$ & 0.1 & 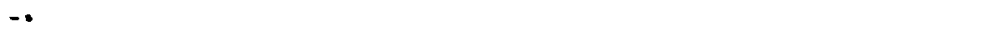 \\
\hline 119.03443 & $\mathrm{C}_{4} \mathrm{H}_{7} \mathrm{O}_{4}$ & 0.8 & \\
\hline 121.05008 & $\mathrm{C}_{4} \mathrm{H}_{9} \mathrm{O}_{4}$ & 1.6 & $\begin{array}{l}\text { sat'd, abundance decreases with time in beam, }\left[G_{122}-\mathrm{HJ} \text { metastable; }-\mathrm{H}_{2,}-\mathrm{H}_{2} \mathrm{O},-\mathrm{CH}_{3} \mathrm{OH} \text { or }\right. \\
-\left(\mathrm{H}_{7}+\mathrm{H}_{2} \mathrm{CO}\right) \mathrm{CAD}:-\mathrm{CH}_{3} \mathrm{OH} \text { or }-\left(\mathrm{H}_{2}+\mathrm{H}_{2} \mathrm{CO}\right)_{,}-\mathrm{H}_{2},-\left(\mathrm{CH}_{3} \mathrm{OH}+\mathrm{H}_{2} \mathrm{CO}\right)\end{array}$ \\
\hline 123.04460 & $\mathrm{C}_{7} \mathrm{H}_{7} \mathrm{O}_{2}$ & 0.3 & \\
\hline 123,00822 & $\mathrm{C}_{6} \mathrm{H}_{3} \mathrm{O}_{3}$ & $<0.1$ & abundance increases with time in beam \\
\hline 124.03717 & $\mathrm{C}_{3} \mathrm{H}_{3} \mathrm{O}_{3}$ & 0.2 & sat'd \\
\hline 125.02387 & $\mathrm{C}_{6} \mathrm{H}_{5} \mathrm{O}_{3}$ & 0.08 & \\
\hline 126.03170 & $\mathrm{C}_{6} \mathrm{H}_{6} \mathrm{O}_{3}$ & 0.07 & \\
\hline 127.03952 & $\mathrm{C}_{6} \mathrm{H}_{7} \mathrm{O}_{3}$ & 0.3 & \\
\hline 127.00313 & $\mathrm{C}_{5} \mathrm{H}_{3} \mathrm{O}_{4}$ & $<0.01$ & \\
\hline 129.05517 & $\mathrm{C}_{6} \mathrm{H}_{9} \mathrm{O}_{3}$ & 0.1 & \\
\hline 129.01878 & $\mathrm{C}_{5} \mathrm{H}_{3} \mathrm{O}_{4}$ & 0.1 & \\
\hline 130.02661 & $\mathrm{C}_{5} \mathrm{H}_{6} \mathrm{O}_{3}$ & 0.1 & \\
\hline 131.03443 & $\mathrm{C}_{3} \mathrm{H}_{7} \mathrm{O}_{4}$ & 0.7 & {$\left[\left(\left\{G_{1 \mathbf{H}_{2}}-2 \mathrm{H}\right\}-\mathrm{H}_{2} \mathrm{O}\right)-\mathrm{H} \Gamma\right.$} \\
\hline 131.07082 & $\mathrm{C}_{6} \mathrm{H}_{11} \mathrm{O}_{3}$ & 0.08 & \\
\hline 133.05008 & $\mathrm{C}_{5} \mathrm{H}_{9} \mathrm{O}_{4}$ & 0.6 & $\left.\left[G_{136}-2 \mathrm{H}-\mathrm{H}\right]\right]^{-} \mathrm{CAD}:-\mathrm{H}_{2} \mathrm{O},-\mathrm{H}_{2} \mathrm{CO},-\mathrm{C}_{2} \mathrm{H}_{4} \mathrm{O}, 73,59$ \\
\hline 135.06573 & $\mathrm{C}_{5} \mathrm{H}_{11} \mathrm{O}_{4}$ & 0.9 & sul'd, $\left[G_{136}-\mathrm{H}^{-}\right.$metastable: $-\mathrm{H}_{2} \mathrm{O},-\mathrm{H}_{2} \mathrm{CAD}:-\mathrm{C}_{2} \mathrm{H}_{4} \mathrm{O},-\mathrm{H}_{2} \mathrm{O}$ \\
\hline 135.02935 & $\mathrm{C}_{4} \mathrm{H}_{7} \mathrm{O}_{5}$ & 0.1 & \\
\hline 137.04500 & $\mathrm{C}_{4} \mathrm{H}_{9} \mathrm{O}_{5}$ & 1.5 & sat $^{\prime} \mathrm{d},\left[\mathrm{HCO}_{2}+\mathrm{Gly}\right]^{-}$ \\
\hline 139.03952 & $\mathrm{C}_{7} \mathrm{H}_{7} \mathrm{O}_{3}$ & 0.1 & \\
\hline 141.05517 & $\mathrm{C}_{7} \mathrm{H}_{9} \mathrm{O}_{3}$ & 0.1 & \\
\hline 141.01878 & $\mathrm{C}_{6} \mathrm{H}_{5} \mathrm{O}_{4}$ & 0.06 & \\
\hline 143.03443 & $\mathrm{C}_{6} \mathrm{H}_{7} \mathrm{O}_{4}$ & 0.2 & \\
\hline 145.05008 & $\mathrm{C}_{6} \mathrm{H}_{9} \mathrm{O}_{4}$ & 0.7 & \\
\hline 149.04500 & $\mathrm{C}_{5} \mathrm{H}_{9} \mathrm{O}_{5}$ & 1.2 & {$\left[G_{152}-2 \mathrm{H}-\mathrm{H}\right], \mathrm{CAD}:-\mathrm{H}_{2} \mathrm{O},-60,-\mathrm{H}_{2} \mathrm{CO}$} \\
\hline 150.05282 & $\mathrm{C}_{3} \mathrm{H}_{10} \mathrm{O}_{3}$ & 2.1 & $\cdots,\left[\mathrm{C}_{2} \mathrm{H}_{2} \mathrm{O}_{2}+\mathrm{Gly}^{\prime}\right]^{\cdot} \mathrm{CAD}:-\mathrm{Gly},-\mathrm{H}_{2} \mathrm{O}$ \\
\hline 151.06065 & $\mathrm{C}_{5} \mathrm{H}_{11} \mathrm{O}_{3}$ & 5.8 & sat'd, $\left[G_{152}-\mathrm{HI}^{-},\left[\mathrm{G}_{1 \mathrm{B2}}-\mathrm{H}_{2} \mathrm{CO}-\mathrm{H}\right]^{-} \mathrm{CAD}:-\mathrm{H}_{2},-60,-\mathrm{CH}_{3} \mathrm{OH}\right.$ or $-\left(\mathrm{H}_{2}+\mathrm{H}_{2} \mathrm{CO}\right),-\mathrm{H}_{2} \mathrm{O}$ \\
\hline 153.05517 & $\mathrm{C}_{\mathrm{B}} \mathrm{H}_{9} \mathrm{O}_{3}$ & 0.1 & \\
\hline 155.05556 & $\mathrm{C}_{4} \mathrm{H}_{11} \mathrm{O}_{6}$ & 0.3 & sat'd \\
\hline 157.05008 & $\mathrm{C}_{7} \mathrm{H}_{9} \mathrm{O}_{4}$ & 0.2 & \\
\hline 159.06573 & $\mathrm{C}_{7} \mathrm{H}_{11} \mathrm{O}_{4}$ & 0.3 & \\
\hline 163.06065 & $\mathrm{C}_{6} \mathrm{H}_{11} \mathrm{O}_{5}$ & 2.6 & {$\left[G_{182}-\mathrm{H}_{2} \mathrm{O}-\mathrm{H}^{-},\left[G_{168}-2 \mathrm{H}-\mathrm{H}^{-},\left[\left[G_{90}-\mathrm{H}_{2} \mathrm{O}\right\}+\mathrm{Gly}-\mathrm{H}\right]^{-} \mathrm{CAD}:\left[G_{90}-\mathrm{H}_{2} \mathrm{O}-\mathrm{HI},-\mathrm{H}_{2} \mathrm{O}\right.\right.\right.$} \\
\hline 164.06847 & $\mathrm{C}_{6} \mathrm{H}_{12} \mathrm{O}_{3}$ & 2.3 & {$\left[G_{90}-\mathrm{H}_{2} \mathrm{O}+\mathrm{Gly}^{-} \cdot \mathrm{CAD}:-\mathrm{H}_{2} \mathrm{O},\left[G_{90}-\mathrm{H}_{2} \mathrm{O}\right]^{-\cdot}\right.$} \\
\hline 165.07630 & $\mathrm{C}_{6} \mathrm{H}_{13} \mathrm{O}_{9}$ & 3.6 & {$\left[G_{165}-\mathrm{H}^{-},\left[G_{74}+\mathrm{Gly}-\mathrm{H}\right]^{-} \mathrm{CAD}:-74,-56,-\mathrm{H}_{2} \mathrm{O},-\mathrm{Gly}\right.$} \\
\hline 171.06573 & $\mathrm{C}_{8} \mathrm{H}_{11} \mathrm{O}_{4}$ & 0.2 & (continued) \\
\hline
\end{tabular}


Table 2-(continued)

\begin{tabular}{|c|c|c|c|}
\hline $\mathrm{m} / \mathrm{z}$ & Formula & Abundance & Comments and Ion Origin \\
\hline 175.06065 & $\mathrm{C}_{9} \mathrm{H}_{11} \mathrm{O}_{3}$ & 0.3 & \\
\hline 177.06065 & $\mathrm{C}_{7} \mathrm{H}_{13} \mathrm{O}_{5}$ & 0.5 & \\
\hline 177.03991 & $\mathrm{C}_{6} \mathrm{H}_{y} \mathrm{O}_{6}$ & 0.2 & \\
\hline 179.05556 & $\mathrm{C}_{6} \mathrm{H}_{11} \mathrm{O}_{6}$ & 1.9 & \\
\hline 180.06339 & $\mathrm{C}_{6} \mathrm{H}_{12} \mathrm{O}_{6}$ & 1.5 & \\
\hline 181.07121 & $\mathrm{C}_{6} \mathrm{H}_{13} \mathrm{O}_{6}$ & 14 & sat'd, $\left[G_{182}-\mathrm{H}^{-},\left[G_{90}+\mathrm{Gly}-\mathrm{H}\right] \mathrm{CAD} ;-\mathrm{H}_{2} \mathrm{O}, 109,-\mathrm{H}_{2} \mathrm{CO},-\mathrm{Gly},-G_{90}\right.$ \\
\hline 183.08686 & $\mathrm{C}_{6} \mathrm{H}_{15} \mathrm{O}_{6}$ & 100 & {$\left[2 \mathrm{Gly}-\mathrm{H}^{-} \mathrm{CAD}:-\right.$ Gly, $-\left(\mathrm{Gly}+\mathrm{H}_{2}\right), 59,-74,-\left(\mathrm{H}_{2}+\mathrm{H}_{2} \mathrm{O}\right)$} \\
\hline 189.07630 & $\mathrm{C}_{8} \mathrm{H}_{13} \mathrm{O}_{5}$ & 0.7 & \\
\hline 191.05556 & $\mathrm{C}_{7} \mathrm{H}_{11} \mathrm{O}_{6}$ & 0.4 & \\
\hline 193.07121 & $\mathrm{C}_{7} \mathrm{H}_{13} \mathrm{O}_{6}$ & 0.6 & \\
\hline 195.08686 & $\mathrm{C}_{7} \mathrm{H}_{19} \mathrm{O}_{6}$ & 1.2 & sat'd alcohol or dimer of [sat'd + unsal'd $-\mathrm{H}^{-}$ \\
\hline 197.10251 & $\mathrm{C}_{7} \mathrm{H}_{17} \mathrm{O}_{6}$ & 0.7 & Gly aggregate, 2 sat'd al cohols or trimer of [2sat'd + unsat' $d-H]^{-}$ \\
\hline 201.07630 & $\mathrm{C}_{9} \mathrm{H}_{13} \mathrm{O}_{5}$ & 0.3 & \\
\hline 209.10251 & $\mathrm{C}_{8} \mathrm{H}_{17} \mathrm{O}_{6}$ & 0.5 & sat'd alcohol or dimer of [sat'd + unsal'd - H] \\
\hline 211.08178 & $\mathrm{C}_{7} \mathrm{H}_{15} \mathrm{O}_{7}$ & 1.1 & sat'd alcohol or dimer of [sat'd + unsat'd $-\mathrm{H}^{-}$ \\
\hline 213.09743 & $\mathrm{C}_{4} \mathrm{H}_{1}, \mathrm{O}_{7}$ & 1.8 & sat'd, Gly aggregate: $\left[G_{122}+\mathrm{Gly}-\mathrm{H}\right]^{-}$ \\
\hline 223.08178 & $\mathrm{C}_{8} \mathrm{H}_{15} \mathrm{O}_{7}$ & 0.5 & Gly aggregate \\
\hline 225.09743 & $\mathrm{C}_{8} \mathrm{H}_{17} \mathrm{O}_{7}$ & 0.8 & Gly aggregate \\
\hline 241.09234 & $\mathrm{C}_{8} \mathrm{H}_{17} \mathrm{O}_{8}$ & 1.0 & sat'd \\
\hline 243.10799 & $\mathrm{C}_{8} \mathrm{H}_{19} \mathrm{O}_{8}$ & 1.8 & sat'd, $\left[G_{152}+G l y-I I\right]^{-}$, metastable: $-G l y,-60$ \\
\hline 255.10799 & $\mathrm{C}_{9} \mathrm{H}_{19} \mathrm{O}_{8}$ & 1.3 & sat'd \\
\hline 256.11582 & $\mathrm{C}_{9} \mathrm{H}_{20} \mathrm{O}_{8}$ & 1.5 & - , sat'd, $\left[G_{90}-\mathrm{H}_{2} \mathrm{O}+2 \mathrm{Gly}^{\prime}\right]^{-} \mathrm{CAD}:-73,-\mathrm{Gly},-(73+\mathrm{Gly}),\left[G_{90}-\mathrm{H}_{2} \mathrm{O}\right]^{-}$ \\
\hline 257.12364 & $\mathrm{C}_{9} \mathrm{H}_{21} \mathrm{O}_{\mathrm{B}}$ & 1.5 & 2 sat'd alcohols \\
\hline 273.11856 & $\mathrm{C}_{9} \mathrm{H}_{21} \mathrm{O}_{9}$ & 2.6 & sat'd, $\left[G_{182}+\mathrm{Gly}-\mathrm{H}\right]^{-}$, metastable: $-\mathrm{Gly}-90$ \\
\hline 275.13421 & $\mathrm{C}_{4} \mathrm{H}_{23} \mathrm{O}_{4}$ & 15 & {$[3 \mathrm{Gly}-\mathrm{H}]$} \\
\hline 303.12912 & $\mathrm{C}_{10} \mathrm{H}_{23} \mathrm{O}_{10}$ & 0.6 & \\
\hline
\end{tabular}

Finally, the first three letters of the matrix (unitalicized) are used to denote an intact matrix molecule.

We now present a theory of background ion formation that builds on the early proposal of Field [24]. The theory has as its basis a large data set, including the extensive list of background ions obtained in this study. For a matrix, Mat, with a molecular weight of w, there are at least four processes involved in the formation of background ions:

Bond fissure leads to matrix-derived free radicals:

$$
\begin{gathered}
\text { Mat }_{w} \rightarrow \cdot M_{x}+\cdot M_{w-x} \\
\text { Mat }_{w} \rightarrow-M_{y}+\cdot M_{w-y} \text { etc. }
\end{gathered}
$$

Coupling forms new molecules:

$$
\begin{gathered}
M_{x}+\cdot M_{y} \rightarrow M_{x+y} \\
\cdot M_{x}+\cdot M_{w-y} \rightarrow M_{x+w-y} \\
\cdot M_{y}+\cdot M_{w-x} \rightarrow M_{y+w-x} \\
\cdot M_{w-x}+\cdot M_{w-y} \rightarrow M_{2 w-x-y} \text { etc. }
\end{gathered}
$$

Losses of neutrals produce lower mass species:

$$
\begin{aligned}
M & \rightarrow\{M-\mathrm{mN}\}+\mathrm{mN} \\
\text { Mat } & \rightarrow\{\text { Mat }-\mathrm{mN}\}+\mathrm{mN}
\end{aligned}
$$

Aggregation/condensation leads to higher mass species:

$$
\begin{aligned}
& M+\text { nMat } \rightarrow[M+\text { nMat }] \\
& \{M-\mathrm{mN}\}+\mathrm{nMat} \rightarrow[\{M-\mathrm{mN}\}+\mathrm{nMat}] \\
& \{\mathrm{M}-\mathrm{mN}\}+\mathrm{nMat} \rightarrow \mathrm{H}_{2} \mathrm{O}+ \\
& {\left[\left((M-m N+M a t)-H_{2} O\right\}+n-1 M a t\right]} \\
& \{\text { Mat }-\mathrm{mN}\}+\mathrm{nMat} \rightarrow[\text { \{Mat }-\mathrm{mN}\}+\text { nMat }] \\
& \left.\left[\{\text { (Mat }-\mathbf{m N}+\text { Mat })-\mathrm{H}_{2} \mathrm{O}\right\}+\mathrm{n}-1 \text { Mat }\right] \\
& \text { Frag + nMat } \rightarrow[\text { Frag }+ \text { nMat }]
\end{aligned}
$$

$\mathrm{N}$ is a stable neutral molecule such as $\mathrm{H}_{2} \mathrm{O}, \mathrm{H}_{2} \mathrm{CO}$, and $\mathrm{CH}_{3} \mathrm{OH}, \mathrm{m}$ and $\mathrm{n}$ are integers $1,2,3, \ldots$, and Frag is a positive or negative matrix fragment ion including odd-electron radical ions. Square brackets and parentheses are used conventionally as grouping symbols, and braces are used to indicate species that may be covalent (e.g., $M-\mathrm{mN}$ is likely covalent).

Losses of neutral molecules to produce lower mass species are presented as occurring prior to ionization, 
but fragmentations driven by the addition or removal of a proton also occur. Positive molecules or aggregates are produced by the addition of a proton or a metal ion, if metal ions are present. Negative ions arise by removal of a proton or by electron capture; if present, anions may also attach to neutral molecules.

Covalent species arise from decomposition of intact molecules and from acid- (or base-) catalyzed condensations occurring between a protonated (or deprotonated) matrix molecule and a suitable reaction partner, such as a carbonyl-containing molecule formed by a bombardment-induced dehydration reaction. In addition, attack on a protonated carbonyl by the oxygen or sulfur atom of a matrix molecule may occur to produce a covalent species with an ether linkage.

Noncovalent species arise from the loose association of matrix molecules with the covalent molerules. Thus, noncovalent ions are proton-bound species (positive ions) or anion-neutral complexes (negative ions).

In addition to producing free radicals by shredding chemical bonds, interaction of the fast-atom or ion with matrix molecules is proposed to produce ionic fragments. Solvation of these fragments, both positive and negative, also leads to background ions (eq 14). If the fragments are acidic, as for many radical cations, or basic, as for alkoxide ions, then the fragments become sources or sinks for protons. Indeed, background ions such as $[\text { Frag } \cdots \mathrm{nM}]^{+}$carry a signature of the ionization chemistry [26], and the study of matrix background ions led to the identification of those acidic reactive fragments that were proposed by Sunner et al. $[27,28]$ and Todd [29].

\section{Results and Discussion}

The results of this study are best discussed in terms of the changes that occur at the molecular level in the region of particle impact, and we elaborate on the description of the bombardment event presented by Sunner et al. [27, 28, 30]. Interaction of the kiloelectronvolt-energy particles is principally with the nuclei of incident molecules [31-33], which are constrained by neighboring molecules. The penetrating atom (or ion), at least initially, interacts indiscriminately with molecules in its path, producing bond rupture, ionization, and internal excitation. Matrix-derived free radicals form, and concomitant momentum transfer prevents immediate recombination and allows for cross coupling. If the motion is decreased by cooling the matrix with a low-temperature probe, then free-radical production is increased, and the abundances of the background ions relative to the protonated matrix molecule increase along with the production of low-mass fragment ions [30, 34, 35]. Thus, the formation of the coupling products proposed by Field [24] can be viewed as an unavoidable consequence of bombardment, just as free-radical and solvated-electron production is a consequence of radiolysis with $\gamma$-rays or high-energy electrons [36].
In addition to bond rupture and excitation, the penetrating atom mechanically dislodges matrix (and analyte) molecules to produce a cavity containing dense, gas-phase material $[9,27,28]$. Thus, the matrix chemistry may be viewed as occurring at local points on the matrix surface. On the basis of previous discussions of FAB ionization $[9,24-30,34,35]$, we propose that these cavities are comprised of inter alia freed electrons, matrix fragment ions, matrix-derived radicals, and analyte and matrix molecules. The chemistry resembles that which occurs within a chemical ionization source except the density of the products is higher. This view is different from that of a general sputtering or peeling away of monolayers of the surface. Any products of the chemistry that remain at the local region will not be sampled again by another impact event for a time on the order of hundreds of milliseconds, calculated for a flux of $10^{10}$ particles $/ \mathrm{s} \cdot \mathrm{cm}^{2}$ and an impact area of $31 \mathrm{~nm}^{2}$ (see below). This time is short for diffusion to be important but is of the same order of magnitude as that required for convection to be important, $4 \mu / \min (\sim 70 \mathrm{~nm} / \mathrm{s})$, which is based on the evaporation rate of glycerol [37]. Products less volatile than glycerol, especially hydrophilic polyhydroxy products and others formed deep within the cavity, may accumulate by not escaping although they may not be observed until their concentration increases as the matrix dries out.

Glycerol is a commonly used matrix and, therefore, the focus of this article. We will show that nearly all matrix ions from glycerol that are greater than $0.1 \%$ relative abundance are in accord with the theory. Table 1 contains a list of selected glycerol positive background ions, and Table 2 is a list of negative background ions. A more complete list of pusitive background ions is available as supplementary material.

\section{Free Radicals}

Homolytic cleavage of $\mathrm{C}-\mathrm{C}$ and $\mathrm{C}-\mathrm{H}$ bonds of a glycerol molecule leads to the formation of $\cdot G_{31}, \cdot G_{61}$, and $\cdot G_{91} C$-centered free radicals. Cleavage of $\mathrm{C}-\mathrm{O}$ and $\mathrm{U}-\mathrm{H}$ bonds also occurs, although to a lesser extent [25], to form $C$-centered $\cdot G_{75}$ and $O$-centered .$O G_{91}$ radicals (Scheme I). The matrix-derived radicals transfer $\cdot \mathrm{H}$ to form unsaturated molecules, or abstract . $\mathrm{H}$ to form small saturated molecules (Figure 1).

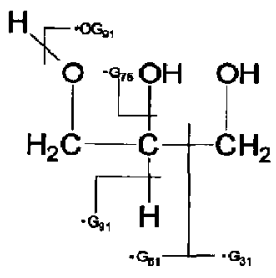

Scheme I 
<smiles>O=CCO</smiles><smiles>O/C=C/O</smiles><smiles>OCCO</smiles><smiles>OCCCO</smiles><smiles>O=CCCO</smiles>

$\mathrm{G}_{76}$ $\mathrm{G}_{74}$<smiles>CC(=O)CO</smiles>

$$
\begin{gathered}
\text { (Gly } \left.-\mathrm{H}_{2} \mathrm{O}\right) \\
\mathrm{G}_{74}
\end{gathered}
$$

Figure 1. Molecules formed by transfer or abstraction of $\cdot \mathrm{H}$ by the glycerol-derived free radicals, and the $\mathrm{H}_{2} \mathrm{O}$-loss decomposition products of glycerol. It is likely that structural isomers also oscur.

\section{Fre--Radical Coupling To Give Molecules and Ions}

The glycerol-derived free radicals couple to produce new molecules (Figure 2). This coupling provides a chemical foundation for the occurrence of four, five, and six carbon low-mass background ions.<smiles>OCC(O)C(O)C(O)C(CO)CO</smiles>

$$
G_{106}: G_{75}-G_{31} \quad G_{122}: G_{91}-G_{31} \quad G_{122}: G_{51}-G_{61}
$$<smiles>OCC(O)C(CO)CO</smiles><smiles>OCC(O)C(O)(CO)CO</smiles>

$$
G_{136}: G_{75}-G_{61}
$$

$$
G_{152}: G_{61}-G_{91}
$$<smiles>OCC(CO)C(O)(CO)CO</smiles><smiles>OCC(O)(CO)C(O)(CO)CO</smiles>

$$
\mathrm{G}_{166}: \mathrm{G}_{91}-\mathrm{G}_{75}
$$

$$
\mathrm{G}_{182}: \mathrm{G}_{91} \cdot \mathrm{G}_{91}
$$

Figure 2 Coupling products produced as a result of coupling the glycerol-derived free radicals produced according to Scheme I. The two radicals bonded to produce each coupling product are listed under each structure.

The coupling products are ionized (e.g., protonated or deprotonated) to give the most abundant background ions observed in the positive and negative ion spectra of glycerol (Tables 1 and 2). The occurrence of these ions bears on the long-standing debate of whether FAB-produced ions are preformed or result from gasphase ionization. Because the coupling products are not preformed, the resulting ions are not preformed. Thus, ionization, at least of the bombardment products, must occur in parallel with (or subsequent to) their formation.

To establish further the occurrence of bombardment-induced free-radical chemistry, consider an example in which stable free radicals from an analyte couple with those from the matrix, in a manner that is analogous to the chemistry that occurs in the neat matrix. Upon bombardment, the herbicide atrazine loses $\cdot \mathrm{C} 1$ to produce a ring-stabilized $\cdot A_{180}$ radical (Scheme II). The principal reaction of this radical is to abstract $\mathbf{H}$ to form $A_{180}-\mathrm{H}$, which, upon protonation, becomes the most abundant sample-specific ion (Table 3 ). Tandem mass spectrometry confirms that the ion of $m / z 182,\left[A_{180}-\mathrm{H}+\mathrm{H}\right]^{+}$, is not a fragment of the protonated molecule. Analogous free-radical mechanisms were reported by others to account for bombardment-induced halogen substitution [38-40].

In addition to $\cdot \mathrm{H}$ abstraction, $\cdot A_{180}$ couples with glycerol-derived radicals (Table 3 ). The analytical implications are that unexpected sample-specific ions are produced. This example underscores the importance of understanding matrix-analyte chemistry. For the matrix hydroxyethyl disulfide, the especially stable Scentered free radical $\cdot \mathrm{SCH}_{2} \mathrm{CH}_{2} \mathrm{OH}$ is produced, and the protonated coupling product $\left[\mathrm{HOCH}_{2} \mathrm{CH}_{2} \mathrm{~S}-A_{180}\right.$ $+\mathrm{H}]^{+}$is comparable in abundance to [atrazine $\left.+\mathrm{H}\right]^{+}$ [18].

\section{Neutral Losses from the Coupling Products}

The molecules formed by free-radical coupling may serve as precursors to other species. Charge-driven fragmentations and/or thermallike decompositions occuring prior to ionization produce background ions lower in mass with elemental compositions that differ from those of the coupling products by the neutral that is lost.

The structures of the protonated and deprotonated coupling products along with their glycerol aggregates, generated in situ from the neat matrix, were investigated by using MS/MS. One feature of struc-<smiles>CCNc1nc(Cl)nc(NC(C)C)n1</smiles>

Scheme II 
Table 3. List of ions and abundances in the FAB spectrum of atrazine in glycerol

\begin{tabular}{|c|c|c|}
\hline $\mathrm{m} / 2$ & $\begin{array}{l}\% \text { Relative } \\
\text { Abundance }\end{array}$ & Ion Origin \\
\hline 93.0552 & 100.0 & 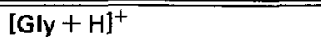 \\
\hline 182.1406 & 28.0 & {$\left[A_{180}-\mathrm{H}+\mathrm{H}\right]^{+}$} \\
\hline 212.1511 & 1.4 & {$\left[A_{180}-G_{31}+\mathrm{H}\right]^{+}$} \\
\hline 215.0938 & 1.4 & [Atrazine] $^{\dagger}$ \\
\hline 216.1016 & 6.3 & [Atrazine $+\mathrm{H}]^{+}$ \\
\hline 242.1617 & 1.0 & {$\left[A_{180}-G_{61}+\mathrm{H}^{+}\right.$} \\
\hline 244.1773 & 2.1 & {$\left[A_{180}-\mathrm{H} \cdots \mathrm{H} \cdots G_{62} \mathrm{~L}\right]^{+}$} \\
\hline 272.1723 & 1.7 & {$\left[A_{180}-G_{91}+H\right]^{+}$} \\
\hline 274.1879 & 3.9 & {$\left[A_{180}-\mathrm{H} \cdots \mathrm{H} \cdots \mathrm{Glyl}^{+}\right.$} \\
\hline 308.1489 & 0.4 & [Alrazine $\cdots \mathrm{H} \cdots$ Gly] $^{+}$ \\
\hline
\end{tabular}

The ion origins were established by using accurate mass measurements and MS MS.

ture that can be determined by MS/MS is the distinction of loosly bound species from those that are completely covalent. Noncovalent adducts, such as proton-bound dimers, decompose metastably by parallel losses of one or the other of the components with the relative abundances being determined by each component's proton affinity [41]. Covalent ions, on the other hand, fragment by low-energy rearrangements and simple-cleavage reactions, leading often to the losses of small, stable molecules such as $\mathrm{H}_{2} \mathrm{O}, \mathrm{NH}_{3}$, and $\mathrm{CO}_{2}$ [42]. The abundance of product relative to precursor ion is generally lower than those from CA of loosly bound adducts. In addition, the kinetic energy release accompanying dissociation, which is observable in the peak widths of the product ions, is greater for covalently bound than for loosely bound ions.

Source-praduced background ions were selected with high resolving power and then allowed to undergo either metastable ion dissociation or CAD. The resulting fragmentation should parallel that occurring in the source region. Therefore, the ions of the species listed in Figure 2 are considered first-generation background ions, and the source-produced ions formed from them by loss of one or more neutrals, either preceding or following ionization, are considered second- or third-generation background ions. The CAD spectra of these latter background ions were used to determine more rigorously the fragmentation chemistry that formally connects the ionized coupling products to background ions lower in mass. Many of the glycerol background ions of $m / z<180$ are the ionized coupling products and their fragments (or their subsequently ionized neutral decomposition products).

Positive ions. The protonated coupling products fragment principally by consecutive losses of $\mathrm{H}_{2} \mathrm{O}$, giving product ions that are increasingly unsaturated. The spectra of $\left[G_{152}+\mathrm{H}\right]^{+}$and $\left[G_{166}+\mathrm{H}\right]^{+}$(Figures 3a and $4 \mathrm{a})$ are representative. The abundant high-mass product ions indicate that the molecule ions are largely
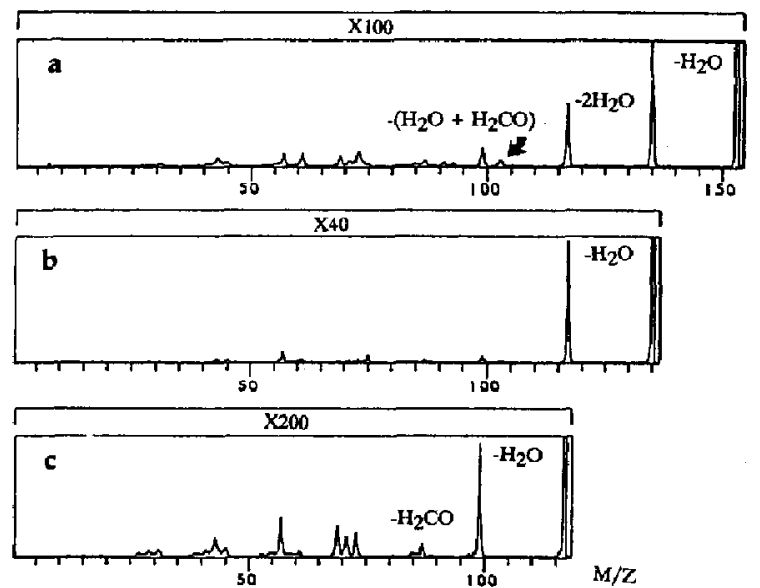

Figure 3. The CAD spectra of the source-produced glycerol background ions (a) $\left[G_{152}+\mathrm{H}\right]^{+}$, (b) $\left[G_{152}-\mathrm{H}_{2} \mathrm{O}+\mathbf{H}\right]^{+}$, and (c) $\left[\mathrm{G}_{152}-2 \mathrm{H}_{2} \mathrm{O}+\mathrm{H}\right]^{+}$.

covalent, in accord with the theory. For the protonated coupling products, loss of $\mathrm{H}_{2} \mathrm{CO}$ does not occur until the loss of an $\mathrm{H}_{2} \mathrm{O}$ molecule creates a double bond in the species.

There is a strong possibility that matrix-derived ions of any given mass-to-change ratio are not of a single structure but rather are a mixture of isomers. Structural isomers of the protonated coupling products could arise, for example, by loss of $\mathrm{H}_{2} \mathrm{O}$ from a glycerol proton-bound cluster or by attachment of a glycerol molecule to an ion lower in mass. Consider further the protonated coupling product $\left[G_{166}+\mathrm{H}^{+}\right.$of $\mathrm{m} / \mathrm{z}$ $167.0919\left(\mathrm{C}_{6} \mathrm{H}_{15} \mathrm{O}_{5}\right)$. Customarily, this ion has been assigned as [2Gly $\left.+\mathrm{H}-\mathrm{H}_{2} \mathrm{O}\right]^{+}$. There is, however, no detectable metastable loss of $\mathrm{H}_{2} \mathrm{O}$ from the putative parent, the proton-bound glycerol dimer, $[2 \mathrm{Gly}+\mathrm{H}]^{+}$
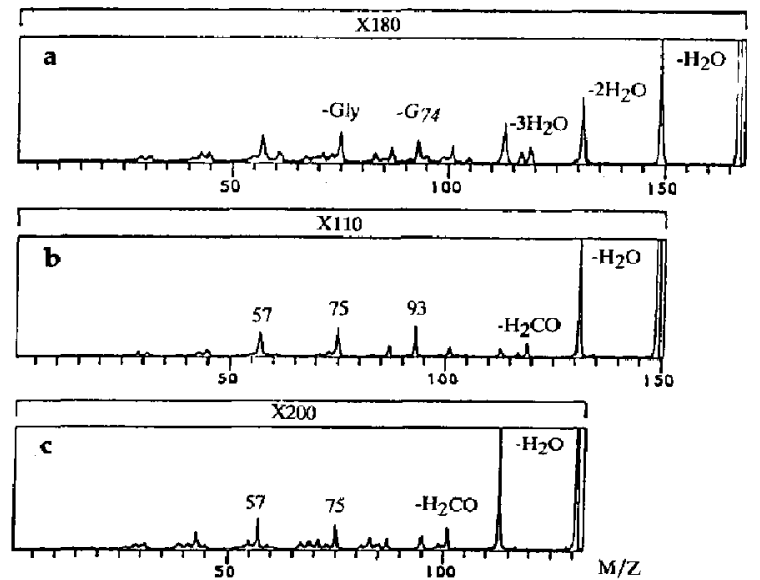

Figure 4. The CAD spectra of the source-produced glycerol background ions (a) $\left[\mathrm{G}_{166}+\mathrm{H}\right]^{+}$, (b) $\left[\mathrm{G}_{166}-\mathrm{H}_{2} \mathrm{O}+\mathrm{H}\right]^{+}$, and (c) $\left[\mathrm{G}_{166}-2 \mathrm{H}_{2} \mathrm{O}+\mathrm{H}^{+}\right.$. 
and collisional activation (CA) produces only a feeble loss of water. Instead, attachment of a glycerol molecule to the ion of $m / z 75.0446\left(\mathrm{C}_{3} \mathrm{H}_{7} \mathrm{O}_{2}\right)$, [ $\mathrm{Gly}-\mathrm{H}_{2} \mathrm{O}+$ $\mathrm{H}]^{+}$and/or to $\left[\mathrm{G}_{74}+\mathrm{H}\right]^{+}$, contributes to the ion population of $m / z 167$; the selected ions lose $G_{74}$ (i.e., Gly $-\mathrm{H}_{2} \mathrm{O}$ ) metastably and both $G_{74}$ and glycerol upon CA (Figure 4a).

The tandem mass spectra of the second- and latergeneration background ions indicate that losses of $\mathrm{H}_{2} \mathrm{CO}$ and, to a lesser extent, of $\mathrm{CH}_{3} \mathrm{OH}$ compete more favorably with consecutive losses of $\mathrm{H}_{2} \mathrm{O}$ (see the comments in Table 1 , and, for representative data, see spectra $B$ and $C$ in Figures 3 and 4). These onecarbon losses formally connect the coupling products to other background ions that at first glance seem unrelated to glycerol or the coupling products (e.g., the ions of $m / z$ 97.0289, 103.0395, and 115.0395, which are listed in Table 1). As for $\left[G_{152}+\mathrm{H}\right]^{+}$and $\left[G_{166}+\mathrm{H}^{+}\right.$, the abundant $\mathrm{H}_{2} \mathrm{O}$-loss product ions formed upon $\mathrm{CA}$ of $\left[G_{152}-\mathrm{nH}_{2} \mathrm{O}+\mathrm{H}^{+}\right.$(Figure $3 \mathrm{~b}$ and $\mathrm{c}$ ) and $\left[G_{166}-\right.$ $\left.\mathrm{nH}_{2} \mathrm{O}+\mathrm{H}\right]^{+}$(Figure $4 \mathrm{~b}$ and $\mathrm{c}$ ) are consistent with these ions having a covalent nature and arising either from in-source fragmentation of $\left[G_{152}+\mathrm{H}\right]^{+}$and $\left[G_{166}\right.$ $+\mathrm{H}^{+}$, respectively, or from thermallike decomposition of $G_{152}$ and $G_{166}$, followed by protonation.

Negative ions. The fragmentation chemistry of the deprotonated or negatively charged coupling products is quite different from that of the protonated ones. Product ions that arise from neutral losses occurring by an $S_{\mathrm{N}} i$ internal nucleophilic displacement mechanism [43] (Scheme III) are of comparable or greater abundance than those arising from $\mathrm{H}_{2} \mathrm{O}$ losses (for representative spectra, see Figure 5). The first- and later-generation negative background ions have a greater propensity to lose $\cdot \mathrm{H}$ and $\mathrm{H}_{2}$ than do the positive ions, both metastably and upon CA, and fragment ions whose formulae are compatible with these losses are more abundant as negative ions than as positive ones (compare Tables 1 and 2). Losses of $\mathrm{H}_{2} \mathrm{CO}$ and $\mathrm{CH}_{3} \mathrm{OH}$ also occur, as for the positive ions.

Source-produced fragmentation and thermallike decomposition of glycerol and the coupling products. Tandem mass spectra can be used to gain insight into the nature of the chemical and physical environment in which an ion is formed and, especially, to provide insight into the formation of background ions. For example, the $[\mathrm{Gly}+\mathrm{H}]^{+}$ions decompose metastably by loss of one

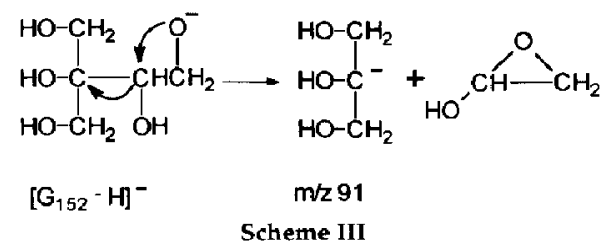

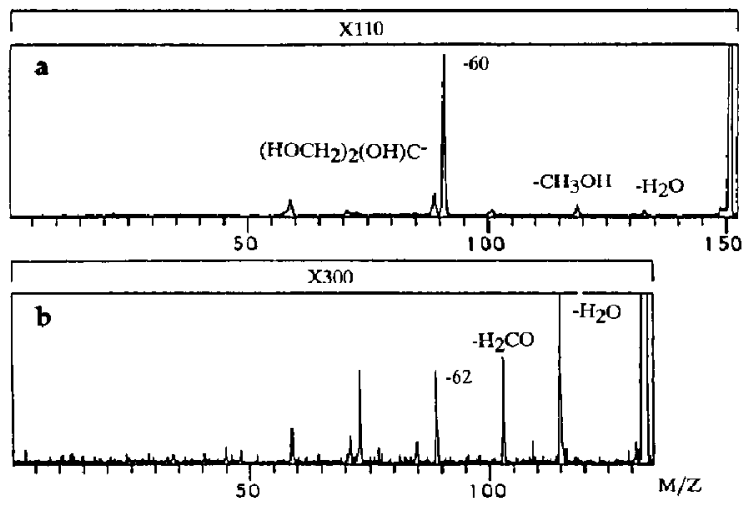

Figure 5. The CAD spectra of the source-produced glycerol background ions (a) $\left[G_{152}-\mathrm{H}\right]^{-}$and (b) $\left[G_{152}-\mathrm{H}_{2} \mathrm{O}-\mathrm{H}\right]^{-}$.

$\mathrm{H}_{2} \mathrm{O}$ molecule to produce a fragment ion of $m / z 75$. The only other fragment ion (that of $m / z 57$ ) distinguishable just above the noise is formed by the loss of two $\mathrm{H}_{2} \mathrm{O}$ molecules, but its abundance is $\mathbf{1 5 0}$ times lower than that of the ion of $m / z$ 75. When the $[\mathrm{Gly}+\mathrm{H}]^{+}$ion is collisionally activated under conditions that give predominantly single collisions $(25 \%$ beam reduction), then the ratio decreases to 7 , whereas the ratio of the source-produced $\mathrm{m} / \mathrm{z} 75$ and 57 ions is 1.4. Either the $[\mathrm{Gly}+\mathrm{H}]^{+}$ions formed in the ion source have more internal energy than those that are collisionally activated or the $m / z 57$ ion has other precursors. For example, acrolein-the principal glycerol thermal decomposition product - is formed and, after protonation, contributes to the population of source-produced $m / z 57$ ions.

Collision-induced fragmentation at the site of bombardment. Locally occurring collisions that produce fragment ions can be envisioned by considering further the environment at the site of impact. Todd and Groenwold [44] found, under conditions that closely match ours, that the loss rate of glycerol is 540 molecules per impact event. If these molecules come from a hemispherical impact cavity [29], then its volume (from the density of glycerol, $1.26 \mathrm{~g} / \mathrm{cm}^{3}$ ) is $65 \mathrm{~nm}^{3}$, and its surface area is $31 \mathrm{~nm}^{2}$. Because the angle between the incoming atom and the surface of the matrix is shallow, however, the cavity is likely to be more troughlike and cylindrical than hemispherical, and a conservative estimate of the depth of the elongated cavity is the calculated radius of the hemisphere, $3.1 \mathrm{~nm}$. This depth is much greater than the dimensions of exiting ions, and for ions formed deep within the cavity, there is no unobstructed exit path, owing to the presence of unremoved matrix molecules. Thus, we suggest that exiting ions may undergo fragment-producing collisions with cavity components, with matrix molecules at the lip of the cavity, and with evaporated and dislodged matrix molecules just outside the cavity. 
These collisions result in an increased abundance of background ions lower in mass than the intact coupling products.

The collision-induced fragmentation scheme is in accord with a report by Kilpatrick et al. [45] who showed that the magnetic-sector, thermospray mass spectra of high-mass analytes produced from early design sources were dominated by fragment ions resulting from collisions occurring during ion drawout from the high-pressure region of ion formation. In addition, Aberth [46] demonstrated that $N_{2}$ gas added to the source region leads to collisions and fragmentations of high-mass ions desorbed by $\mathrm{Cs}^{+}$-ion bombardment.

Thermallike decompositions. Although we are able to demonstrate from the tandem mass spectra that unsaturated positive ions can arise from charge-promoted losses of $\mathrm{H}_{2} \mathrm{O}$ from both glycerol and the coupling products, these may not be the only sources of the ions. Thermallike losses occurring prior to ionization would ultimately lead to ions with identical elemental compositions. In accord with the bombardment event described at the beginning of the Results and Discussion section, matrix molecules that come into intimate contact with the atom beam and those on the periphery of the point of impact that acquire internal energy from the high local temperature are expected to undergo thermallike decompositions (e.g., lose small neutral moleculcs). In addition, concomitant fragmentation and rearrangement losses may occur after free-radical coupling [47].

Glycerol thermally decomposes principally by the loss of two $\mathrm{H}_{2} \mathrm{O}$ molecules to form the conjugated molecule acrolein, whereas hydroxyacetone, acetaldehyde, and formaldehyde are formed in lesser amounts [48]. These carbonyl-containing products react further and condense with glycerol to form acetals and ketals [48]. Experimental evidence for thermallike, bombardment-induced decompositions comes from a study by Lehmann et al. [49] who showed that formaldehyde is produced during bombardment of glycerol and then reacts with the amino group of added oligopeptides to form a Schiff's base. Given the volatility of formaldehyde, this suggests that the environment of the impact cavity must be sufficiently dense that solutionlike chemistry can occur.

The structures of the coupling products given in Figure 2 are similar to that of glycerol, and their decomposition pathways should also be similar to those of glycerol. If thermallike decompositions (e.g., consecutive losses of $\mathrm{H}_{2} \mathrm{O}$ from the neutral coupling product) contribute to the formation of background ions, then the products of those decompositions must become ionized to be seen. It follows that they should appear in spectra of both positive and negative ions. Unfortunately, all of the protonated coupling products undergo charge-promoted $\mathrm{H}_{2} \mathrm{O}$ losses to give abundant $\left[\mathrm{G}-2 \mathrm{H}_{2} \mathrm{O}+\mathrm{H}\right]^{+}$fragment ions, which cannot be distinguished from ions formed by protonation of de- composition products arising from losses of two water molecules from $G$. The deprotonated coupling products, however, produce upon CA only low abundance $\left[\mathrm{G}-2 \mathrm{H}_{2} \mathrm{O}-\mathrm{H}^{-}\right.$fragment ions (compare Figures 3 and 4 with Figure 5). Yet, background ions such as $\left[G_{122}-2 \mathrm{H}_{2} \mathrm{O}-\mathrm{H}\right]^{-}(m / z 85.0289)$ and $\left[G_{152}-2 \mathrm{H}_{2} \mathrm{O}\right.$ $-\mathrm{H}]^{-}(m / z 117.0552)$ are very abundant source-produced ions. In fact, the source-produced ions arising from the loss of two $\mathrm{H}_{2} \mathrm{O}$ molecules from $G_{152}$ are more abundant than those arising from the loss of one (Table 2), which suggests that thermallike decompositions do occur and that the formation of a conjugated system is a driving force. Clearly, thermallike decompositions of coupling products prior to ionization play a role in background ion formation.

Transfer and abstraction of $\cdot H$ by matrix-derized free radicals. We propose that the glycerol-derived free radicals can transfer or abstract $\cdot \mathrm{H}$ to form unsaturated or saturated species $1 \mathrm{u}$ lower or higher in mass than that of the precursor radical. As pointed out by Field [24], - $G_{91}$ can transfer $\cdot H$ to form $G_{90}$, which, upon ionization, appears as a major background ion in both the positive (at $m / z$ 91) and negative (at $m / z$ 89) ion spectra (Tables 1 and 2). Although the positive $\left[G_{90}+\right.$ $\mathrm{H}]^{+}$ion can be formally attributed to loss of $\mathrm{H}_{2}$ from protonated glycerol, neither the metastable ion spectrum nor the CAD spectrum of protonated glycerol shows production of a $m / z 91$ product ion. As for the coupling products, species lower in mass than the products formed by $\mathrm{H}$-atom transfer or $\mathrm{H}$-atom abstraction are produced by loss of stable neutral molecules, such as $\mathrm{H}_{2} \mathrm{O}$ (e.g., see the comments for the ion of $m / z 165.0763$ in Table 1).

The $\cdot G_{75}$ radical abstracts $\cdot H$ to form $G_{76}$ (Figure 1 ), which appears as a protonated species. Further evidence for the formation of $G_{76}$ is the CAD spectrum of the species that corresponds to the proton-bound dimer between $G_{76}$ and glycerol; instead of high mass product ions, only $[\mathrm{Gly}+\mathrm{H}]^{+}$and $\left[\mathrm{G}_{76}+\mathrm{H}\right]^{+}$product ions form (Figure 6).

\section{Aggregation}

The products of single-step, free-radical coupling and their decomposition products account for virtually all of the background ions of $m / z<180$ and with abundances greater than $\sim 0.1 \%$. For other matrices (e.g., 3-nitrobenzyl alcohol), propagated free-radical polymerization may be an important mechanism for producing high-mass background ions [50]. The elemental compositions and the tandem mass spectra of the glycerol background ions, however, are not consistent with the hypothesis that the coupling products combine with glycerol to produce covalent high mass species via continued free radical polymerization. Instead, background ions higher in mass than the coupling products form when intact glycerol molecules (or protonated glycerol) aggregate with ions (or neutral 


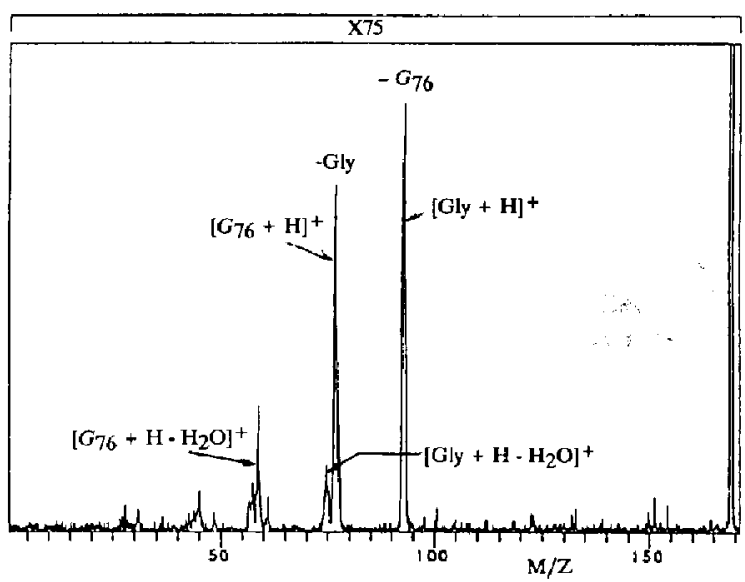

Figure 6. The CAD spectrum of the source-produced protonbound dimer comprised of $G_{76}$ and glycerol $\left[G_{76} \cdots \mathrm{H} \cdots \mathrm{Gly}\right]^{+}$.

species) from the coupling products. Aggregation can occur via $\mathrm{H}$-bonding, charge-dipole interactions, and condensation reactions. Both glycerol and water have large dielectric constants and unusually high boiling points owing to extensive $\mathrm{H}$-bonding. Attachment of glycerol to lower mass ions is analogous to the addition of $\mathrm{H}_{2} \mathrm{O}$ to ionic species in atmospheric pressure ionization mass spectrometry $[51,52]$ and to the consecutive attachment of $\mathrm{H}_{2} \mathrm{O}$ to $\mathrm{H}_{3} \mathrm{O}^{+}$in free-jet-expansion experiments $[53,54]$. Because glycerol is more reactive than water, however, it may also condense with unsaturated molecules and become covalently incorporated.

As a basic premise, aggregation occurs and the products undergo collisional cooling in the dense, gaseouslike region within a cavity produced by bombardment at the matrix surface. Because the residence time of an ion in the cavity is not known precisely, it is not clear whether aggregation occurs primarily after ionization or whether the only aggregate species observed are neutral ones that become ionized subsequently because the residence time of the ions in the cavity is so small. Blakley and Vestal [55] and DePauw [56] suggested that analyte ions and, by analogy, background ions are preformed and arise from declustering of small droplets.

Experimental evidence for aggregation comes from (1) elemental compositions, (2) abundances of background ions separated by $92 \mathrm{u}$, (3) tandem mass spectra, and (4) the rolloff observed in the abundance of background ions on going to higher mass. We present the evidence supporting aggregation, both noncovalent and covalent, in this order.

Elemental compositions. The simplest test of matrix aggregation is the elemental composition of high mass background ions. In Tables 1 and 2 we listed the most abundant of these ions that have masses higher than the molecule ion of $G_{182}$. All have elemental composi- tions that are consistent with the aggregation of neutral glycerol with ions $92 \mathrm{u}$ lower in mass.

Abundances of background ions separated by $92 u$. Graphic evidence for aggregation was obtained by plotting the glycerol spectra in 92-u increments. For both the positive (Figure 7) and negative ion spectra (not shown), there are series of background ions that differ by $92 \mathbf{u}$ (the nominal mass of glycerol) and that have similar abundance patterns (e.g., ions of $m / z 153$, 245,337 , and 429 ).

Tandem mass spectra. The tandem mass spectra of the positive background ions are discussed more fully than those of the negative ions, but the compositions of the negative ions usually parallel those of the positive ions, differing only by two $\mathrm{H}$ atoms.

$[G \cdots H \cdot G l y]^{+}$and $\left[(G \cdots H \cdots G l y)+n G[y]^{+}\right.$. Upon $C A$ of the proton-bound dimers $[G \cdots H \cdots$ $\mathrm{Gly}^{+}$, where $\mathrm{G}$ represents the intact coupling products $G_{106}, G_{122}, G_{136}$, etc. (Figure 2), the $(G+H)^{+}$is produced more readily. In each case, the abundance of the protonated coupling product is five to 25 times greater than that of protonated glycerol, indicating that

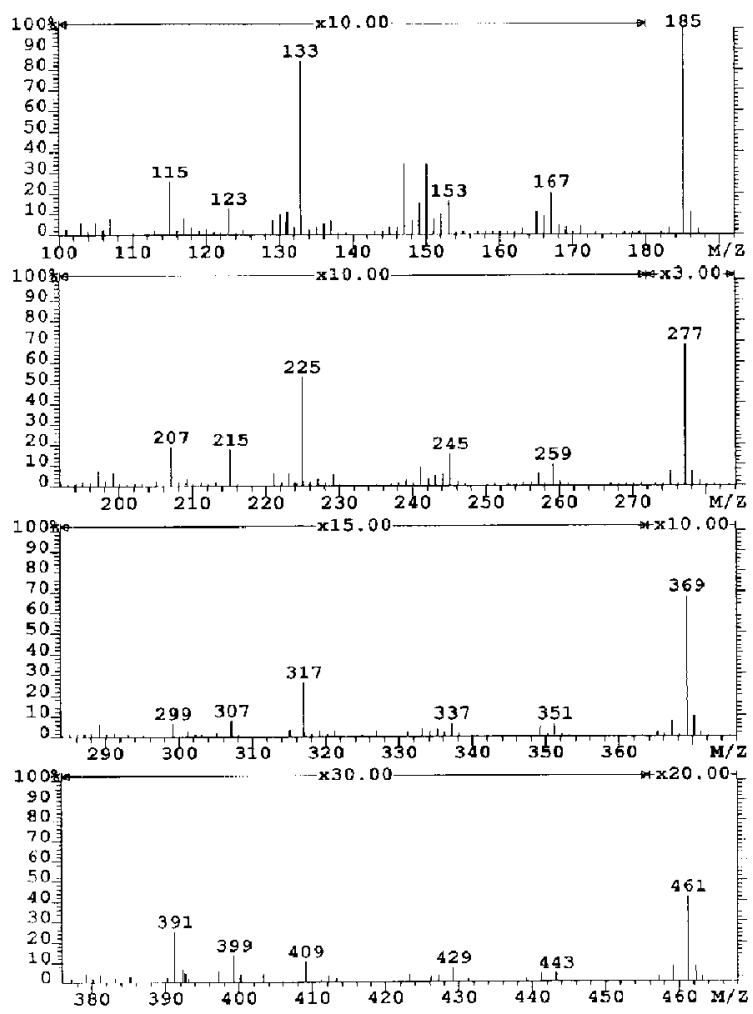

Figure 7. The $\mathrm{Cs}^{+}$liquid secondary ion mass spectrum of glycerol. The $x$-axis for each box is $92 u$, the nominal mass of glycerol. The ions of $m / z \quad 115$ and 133 are not attributed to background ions, but to $\left[\mathrm{Gly}+\mathrm{Na}^{+}\right.$and $[\mathrm{Gly}+\mathrm{Cs}]^{+}$, respertively. See text for discussion. 
the proton affinities of the coupling products are higher than that of glycerol. The higher proton affinity is in accord with the additional hydroxyl groups of the coupling products. Upon metastable ion decomposition, protonated glycerol is only detected, just above the noise, in the spectrum of $\left[G_{106} \cdots \mathbf{H} \cdots G l y\right]^{+}$. This is reasonable given that glycerol has fewer hydroxyl groups than any of the coupling products except $G_{106}$.

$\left[\{G-m N l+n G l y+H]^{+}\right.$and $[\{G l y-m N\}+n G l y$ $+\mathrm{H}^{+}$. The simple attachment scheme becomes complicated for background ions formally corresponding to aggregation of glycerol with unsaturated decomposition products. We focus on one class of aggregates to illustrate how the tandem mass spectra were used to probe structure type.

Consider the $\left[\left(\mathrm{G}-2 \mathrm{H}_{2} \mathrm{O}+\mathrm{H}\right)+\mathrm{nGly}\right]^{+}$oligomers. The background ion of $m / z 209.1025\left(\mathrm{C}_{8} \mathrm{H}_{17} \mathrm{O}_{6}\right)$ has three possible origins. It could form by the attachment of one glycerol molecule to the covalent $\left[G_{152}-\right.$ $2 \mathrm{H}_{2} \mathrm{O}+\mathrm{H}^{+}$ion of $m / z 117.0552(0.5 \%$ abundance) to give $\left[\left(\mathrm{G}_{152}+2 \mathrm{H}_{2} \mathrm{O}\right]+\mathrm{Gly}+\mathrm{H}\right]^{+}$. An alternative origin is loss of two $\mathrm{H}_{2} \mathrm{O}$ molecules from a proton-bound dimer comprised of glycerol and $G_{152},\left[\left(G_{152}\right.\right.$ $\left.\cdots \mathrm{H} \cdots \mathrm{Gly})-2 \mathrm{H}_{2} \mathrm{O}\right]^{+}$. A third possibility is a covalent product formed concomitantly with loss of $\mathrm{H}_{2} \mathrm{O}$, $\left[\left\{\left(G_{152}-\mathbf{H}_{2} \mathrm{O}+\mathrm{Gly}\right)-\mathrm{H}_{2} \mathrm{O}\right\}+\mathbf{H}\right]^{+}$.

The first species, $\left[\left\{G_{152}-2 \mathrm{H}_{2} \mathrm{O}\right\}+\mathrm{Gly}+\mathrm{H}\right]^{+}$, should fragment by competitive losses of the components. Because energy is required to overcome the attractive $\mathbf{H}$-bonding forces, the product ions formed by luss of glycerol and $\left(G_{152}-2 \mathbf{H}_{2} \mathrm{O}\right)$ are expected to be of relatively low abundance in the metastable ion spectrum, as is observed in Figure 8a. The major fragment ions arise from consecutive losses of $\mathrm{H}_{2} \mathrm{O}$ molecules and likely originate from a small population of ions with sufficient internal energy to overcome the barrier to water-loss rearrangement. Upon collisional activation, however, the energy deficit for separation can be overcome, and $\left[G_{152}-2 \mathrm{H}_{2} \mathrm{O}+\mathrm{H}\right]^{+}$, produced from the loss of glycerol, is the major product ion, along with those resulting from $\mathrm{H}_{2} \mathrm{O}$ loss (Figure $8 \mathrm{~b}$ ).

Contribution of the second species, $\left[\left(\mathrm{G}_{152} \cdots \mathrm{H} \cdots\right.\right.$ Gly) $-2 \mathrm{H}_{2} \mathrm{OJ}^{+}$, to the $\mathrm{C}_{8} \mathrm{H}_{17} \mathrm{O}_{6}$ ion population requires that loss of the first of two $\mathrm{H}_{2} \mathrm{O}$ molecules occurs from one of the components while it is protonbound to the other. The metastable ion and CAD tandem mass spectral of $\left[G_{152} \cdots \mathrm{H} \cdots \mathrm{Gly}\right]^{+}$(Figure 8c) contain only low abundance product ions formed by expulsion of water and no detectable product ions arising from losses of two water molecules. In addition, for $[2 \mathrm{Gly}+\mathrm{H}]^{+}$, product ions from loss of one or more stable neutrals, such as $\mathrm{H}_{2} \mathrm{O}, \mathrm{H}_{2} \mathrm{CO}$, and $\mathrm{CH}_{3} \mathrm{OH}$, are formed with low abundances upon $\mathrm{CAD}$ and are not observed at all in metastable ion decompositions. Therefore, fragmentation of the glycerol oligomers and of the oligomers of the coupling products with glycerol is not likely to be a major source of background ions.

The losses of $\mathrm{H}_{2} \mathrm{O}$ from $\left[\left(\mathrm{G}_{152}-2 \mathrm{H}_{2} \mathrm{O}+\mathrm{H}\right)+\right.$ Gly $]^{+}$(Figure $8 b$ ), from other $\left[\left(G-\mathrm{mH}_{2} \mathrm{O}+\mathrm{H}\right)+\right.$
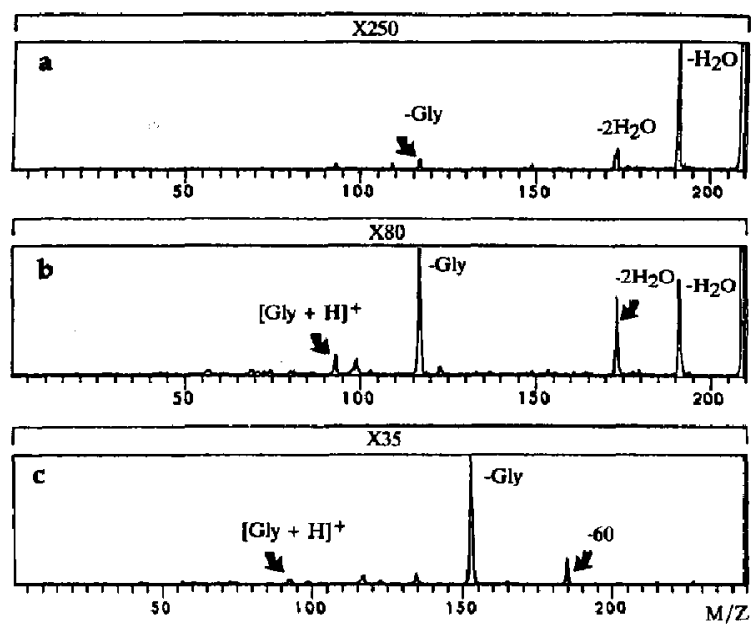

Figure 8. The metastable ion (a) and CAD spectrum (b) of the source-produced glycerol background ion $\left[\left(G_{152}-2 \mathrm{H}_{2} \mathrm{O}+\mathrm{H}\right)\right.$ $+\mathrm{Gly}^{+}(\mathrm{m} / \mathrm{z}$ 209.1025). (c) CAD spectrum of the source-produced glycerol background ion $\left[\left(\mathrm{G}_{152}+\mathrm{H}\right)+\mathrm{Gly}\right]^{+}$.

$\mathrm{Gly}^{+}$species, and from $\left[\left(\mathrm{Gly}-\mathrm{mH}_{2} \mathrm{O}+\mathrm{H}\right)+\mathrm{Gly}^{+}\right.$ (Table 1) suggest that there is, in addition to the loosely bound species that lose a glycerol molecule, a covalent population of ions produced by reactions of unsaturated coupling products and glycerol. By analogy with solution and pyrolysis chemistry of glycerol [48], the products are likely to be acetals or ketals formed from gas-phase acid- or base-catalyzed condensations (for the protonated or deprotonlated oligoniers, respectively) of glycerol with aldehydes or ketones produced as a result of water loss. One possibility is illustrated in Scheme IV.

Glycerol, hydroxyacetone, $G_{74}$, and acrolein, $G_{56}$ are expected to be abundant species at the regions of atom impact. Losses of $\mathrm{H}_{2} \mathrm{O}$ from $\left[\mathrm{G}_{74}+\mathrm{Gly}+\mathrm{H}\right]^{\prime}$ (Figure 4a) and $\left[G_{56}+\mathrm{Gly}+\mathrm{H}\right]^{+}$(Figure $4 \mathrm{~b}$ ) are evidence that solution-phase chemistry has gas-phase analogs and readily account for the high abundance of the background ions of $m / z 149$ and 131 (Table 1). Although these latter ions can be formed by consecutive losses of water from $\left[\mathrm{G}_{166}+\mathrm{H}\right]^{+}$, the ions of $\mathrm{m} / z 149$ and 131 are still more abundant relative to the saturated molecule ion than those from the other coupling products (e.g., compare the abundances of $\left[\mathrm{G}_{152}-\right.$ $\left.2 \mathrm{H}_{2} \mathrm{O}+\mathrm{H}\right]^{+}$and $\left[G_{152}+\mathrm{H}\right]^{+}$with the abundance of the ion of $m / z 131$ and the $\left[G_{166}+\mathrm{H}\right]^{+}$ion). Acyclic hemiketals, the products of the top reaction in Scheme IV, were reported to be formed with glycerol and carbonyl-containing analytes [57]. These species may be the initially formed adduct between a singly dehydrated coupling product and glycerol, and the proposed structures account for the additional covalent character, determined from the CAD spectra, of [( $G-$ $\left.\mathrm{H}_{2} \mathrm{O}+\mathrm{H}\right)+\mathrm{Gly}^{+}$ions.

Additional evidence for the formation of covalent aggregates is the endpoint for glycerol loss from aggregates that were collisionally activated under conditions 
<smiles></smiles><smiles>[R]C([R])(O)OC[C@H](O)CO</smiles><smiles>[R]C([R])([OH2+])OCC(O)CO</smiles>

that produce multiple collisions ( $80 \%$ CAD). In this experiment, all of the loosely attached glycerol molecules should be lost sequentially if the aggregates are noncovalent. As a control, the all-saturated aggregate $\left[\left(G_{152}+H\right)+2 G l y\right]^{+}$was collisionally activated; both glycerol molecules are lost down to the $\left[G_{152}+\right.$ $\mathrm{H}^{+}$ion (Figure 9a). Evidence for the presence of more covalent components of the ion population, therefore, would be early termination of glycerol loss.

We address two examples of unsaturated glycerolaggregate background ions. The first is the ion of $m / z$ 315 , which corresponds to noncovalent aggregation of two glycerol molecules with the covalent source-produced ion of $m / z 131$, which was described previously as being composed of two ion populations: the decomposition product $\left[\mathrm{G}_{166}-2 \mathrm{H}_{2} \mathrm{O}+\mathrm{H}\right]^{+}$and the protonated acetal formed by the condensation of glyc-
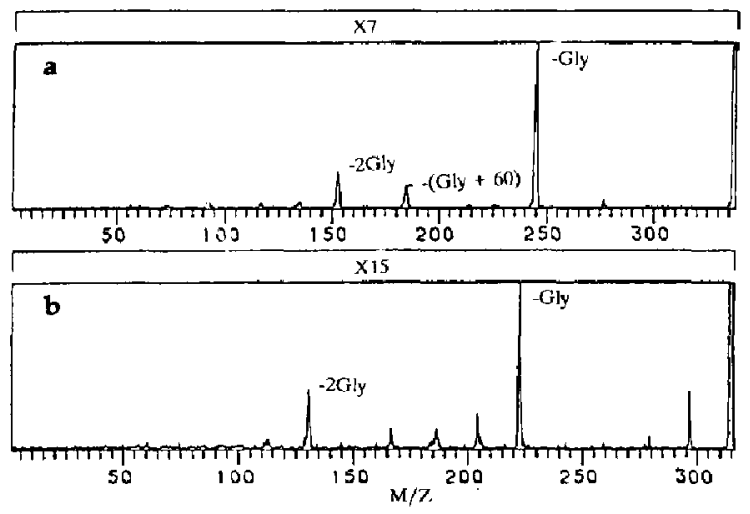

Figure 9. The CAD spectra $(80 \%$ beam reduction) of the source-produced glycerol background ions (a) $\left[\left(G_{152}+H\right)+\right.$ $2 \mathrm{Gly}]^{+}$and (b) $[131+2 \mathrm{Gly}]^{+}$, where 131 is the background ion of $m / z 131.0708$, which corresponds to both $\left[G_{166}-2 \mathrm{H}_{2} \mathrm{O}+\right.$ $\mathrm{H}]^{+}$and $\left[\left(\mathrm{G}_{56}+\mathrm{Gly}+\mathrm{H}\right)-\mathrm{H}_{2} \mathrm{O}\right]^{+}$. erol with acrolein, both of which are covalent. Upon CA of the aggregate ion, a product ion of $m / z 131$ is formed by losses of both glycerol molecules, and the relative abundances of the consecutive glycerol-loss product ions parallel those in the control spectrum (compared to the spectra in Figure 9). In addition to losses of glycerol, there is a relatively abundant $\mathrm{H}_{2} \mathrm{O}$ loss product ion, which we assign to the acid-catalyzed condensation of $\left\{G_{166}-2 \mathrm{H}_{2} \mathrm{O}\right\}$ with one of the glycerol solvent molecules. Of the two ion populations, the aggregate with the ring-containing acetal should more readily lose both glycerol molecules, and we attribute the relatively abundant product ion of $\mathrm{m} / z 131$ primarily to the protonated acetal.

The second example is the series of $\left[\left(G_{152}-\mathrm{mH}_{2} \mathrm{O}\right.\right.$ $+\mathrm{H})+2 \mathrm{Gly}^{+}$ions. Loss of one glycerol gives rise to a major fragment ion, but loss of a second glycerol occurs to only a minor extent (Figure 10), unlike for the previous example. This can be accounted for by proposing that a glycerol molecule is chemically aggregated with the $\left[G_{152}-\mathrm{mH}_{2} \mathrm{O}+\mathrm{H}\right]^{+}$ion by condensation of glycerol with the $\left[\mathrm{G}_{152}-(\mathrm{m}-1) \mathrm{H}_{2} \mathrm{O}+\mathrm{H}\right]^{+}$ species $18 \mathrm{u}$ higher in mass, analogous to the condensation of glycerol with acrolein in the previous example. For example, consider the $\left[\left(G_{152}-\mathbf{H}_{2} \mathrm{O}+\mathrm{H}\right)+\right.$ $\mathrm{Glyl}^{+}$species. Chemical aggregation of glycerol can also occur by the formation of an ether linkage (to produce the hemiketal) by attack on the protonated carbonyl (top half of Scheme IV).

On the basis of tandem mass spectra, we conclude that background ions of the type $\left[\left(G-\mathrm{mI}_{2} \mathrm{O}+\mathrm{H}\right)+\right.$ nGly $]^{+}$are comprised of both noncovalent and covalent ion populations. For all of the coupling products, the abundances of the source-produced $\left[\left(G-\mathrm{mH}_{2} \mathrm{O}\right.\right.$ $+\mathrm{H})+\mathbf{n G l y}^{+}$ions $(\mathbf{n}>2)$, however, are low. If glycerol reacts subsequently with the unsaturated, covalent aggregates, then it is only to a minor extent.

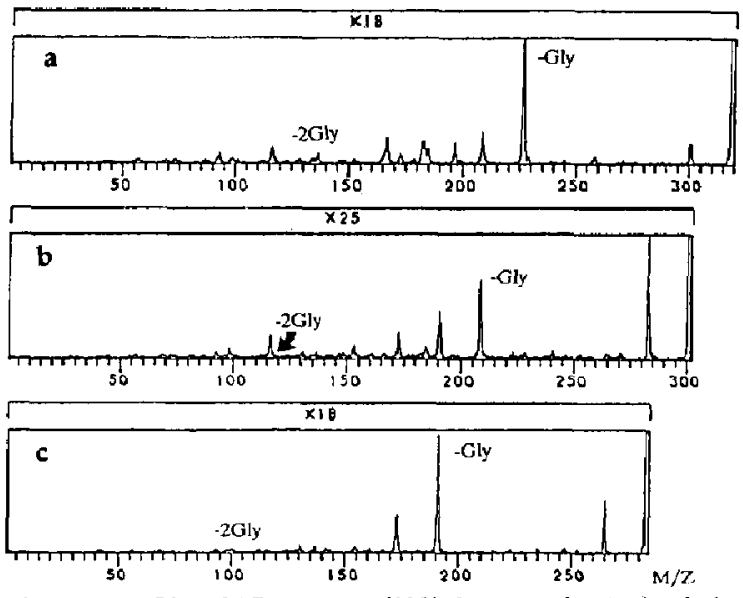

Figure 10. The CAD spectra ( $80 \%$ beam reduction) of the source-produced glycerol background ions (a) $\left[\left(\mathrm{G}_{152}-\mathrm{H}_{2} \mathrm{O}+\right.\right.$ $\mathrm{H})+2 \mathrm{Gly}]^{+}$, (b) $\left[\left(\mathrm{G}_{152}-2 \mathrm{H}_{2} \mathrm{O}+\mathrm{H}\right)+2 \mathrm{Gly}\right]^{+}$, and (c) $\left[\left(\mathrm{G}_{152}\right.\right.$ $\left.\left.+3 \mathrm{H}_{2} \mathrm{O}+\mathrm{H}\right)+2 \mathrm{Cly}\right]^{+}$. 
Therefore, background ions higher in mass than approximately $400 \mathrm{u}$ are predominantly clusters held together by noncovalent bonds. This is in accord with the results of Cox et al. [58] for liquid secondary ion mass spectra obtained by using an ion trap mass spectrometer. High-mass background ions are reported to be dissociated within the relatively high-pressure confines of the trap.

Rolloff in abundance. There is a rolloff in the background ion abundance on going from low mass to approximately $500 \mathrm{u}_{r}$ and then a gradually decreasing baseline continuing to the highest mass ions that can be transmitted by the spectrometer. This occurs not only for glycerol but also for other FAB matrices. The rolloff can be rationalized by proposing that the highmass background ions consist of a charged core comprised of the most basic or acidic component of the aggregate. This charged core is a protonated or depro tonated coupling product, decomposition product, or covalent aggregate, for both the positive and negative ions. Attachment of matrix molecules occurs subsequently via charge-dipole and $\mathrm{H}$-bonding interactions. Because the latter interactions have a strong dependence on the distance from the charged center, the interaction between the core ion and the matrix molecules comprising the first solvation shell is strongest. Beyond the first solvation shell, which is likely comprised of at most five or six matrix molecules, there is a leveling in the strength of the intermolecular interactions as $\mathbf{H}$-bonding and van der Waals forces become the principal modes of interaction. 'I'hus, there is a rolloff for matrix ions of $\mathrm{m} / \mathrm{z}>\sim 500$ the point at which the first solvation shell becomes filled. Moreover, aggregation of more and more matrix molecules is increasingly entropy demanding. These considerations may also apply to the background ions formed by matrix-assisted laser desorption, which are abundant below approximately $400 \mathrm{u}$ and decrease for increasing mass (see the spectra in refs 59-61).

\section{Other Matrices and Examples of Matrix Analyte Chemistry}

We investigated the bombardment-induced matrix chemistry of thioglycerol, DTT/DTE and hydroxyethyl disulfide (HEDS). The chemical changes occurring in these matrices are more complex than in glycerol, but a few general statements can be made. For thioglycerol and DTT/DTE matrices, loss of $\mathrm{H}_{2} \mathrm{O}$ from the protonated molecules and selected background ions is more facile than loss of $\mathrm{H}_{2} \mathrm{~S}$. For HEDS, the $\cdot \mathrm{SCH}_{2} \mathrm{CH}_{2} \mathrm{OH}$ radical is produced abundantly and reacts with other matrix-derived radicals as well as with analyte-derived radicals such as the $\cdot A_{180}$ radical from atrazine [18]. As for thioglycerol and DTT/DTE, the background ions from HEDS appear to retain the sulfur heteroatom because they are shifted significantly to lower mass than are isobaric ions that do not contain sulfur, owing to the negative mass defect of sulfur. This has important ramifications for trace analysis by FAB-MS [17].

During the completion of this manuscript, Tuinman and Cook [62] reported that glycerol-derived free radicals react with surfactant-derived free radicals produced by $\mathrm{H}$-atom abstraction, in accord with the matrix chemistry presented here and elsewhere [18]. We have also observed such adducts for the analysis of surfactants, not only for glycerol but also for the HEDS matrix.

\section{Suppression of Background Ions by Surface Active Analytes}

If a surface active analyte is present, then the evaporation and sputtering rate of glycerol is diminished [20], yet the abundance of background ions is observed to decrease. This can be understood by proposing that the analyte serves the role of a buffer, slowing the atom as it traverses the surface layer of analyte molecules. This notion is supported by the observation that the absolute abundances of background ions increase with increasing primary ion energy [21, 63]. The minimum level of a surface-aclive sample that would produce a suppression effect can be calculated by assuming that the number of molecules in the bulk of the matrix is negligible until the number of molecules exceeds that required to just cover the surface (Table 4). The surface area of the matrix liquid is approximately the area of the probe tip, which in our studies was $13 \mathrm{~mm}^{2}-$ a typical value. The cross-sectional area for saturated fatty acids, a representative analyte, is $\sim 20 \AA^{2} /$ molecule [64]. Therefore, the number of fatty acid molecules needed to just form a monolayer at the liquid surface is $6.5 \times 10^{13}$ or $100 \mathrm{pmol}$. All of the concentrations should be considered as lower limits for producing surface coverage effects because the actual number of molecules (especially peptide molecules) at the surface will be less because some will be distributed in the bulk before the surface is covered. Thus, for analyses at the level listed in the table and lower, the analyte

Table 4. Formal analyte concentration and number of moles necessary to form a surface monolayer in a matrix distributed as a film on a probe tip with a surface area of $13 \mathrm{~mm}^{2}$

\begin{tabular}{lcc}
\hline \multicolumn{1}{c}{ Analyte } & $\begin{array}{c}\text { Formal } \\
\text { Concentration } \\
\text { in the matrix }\end{array}$ & $\begin{array}{c}\text { Number of } \\
\text { Molecules } \\
\text { (pmol) }\end{array}$ \\
\hline \hline Fatty acids & $5.5 \times 10^{-5} \mathrm{~F}$ & $100(\sim 28 \mathrm{ng})$ \\
Phosphoglycer(des & $2.1 \times 10^{-5} \mathrm{~F}$ & $54(\sim 42 \mathrm{ng})$ \\
Peptides & $5.0 \times 10^{-6} \mathrm{~F}$ & $10(\sim 15 \mathrm{ng})$
\end{tabular}

The cross-sectional areas for saturated fatty acids, $20 \AA^{2}$, and for phospholipids, 40 $\AA^{2}$. (phosphatidylchalines containing saturated fatty acids) were obtained from ref 64 . The cross-sectional area for peptides, $100 \AA^{2}$, is an estimate. The concentration is based on the use of $\mathbf{2} \mu \mathrm{L}$ of matrix, and the mass of the analyte was calculated from the molecular weights of representative molecules. See text. 
can rightly be considered a small perturbation to the matrix liquid, and suppression effects due to surface coverage, unfortunately, will not be important, especially for hydrophilic molecules.

\section{Background Ions in Continuous-Flow FAB}

A variant of static FAB-MS is dynamic or continuousflow FAB-MS (CF-FAB) [65], in which an aqueous or methanolic solution of glycerol is flowed continuously onto the probe tip. As a result of a dynamic steady state, a film of liquid remains on the tip. Because water is rapidly removed from glycerol in high-vacuum [66], we propose that the remaining liquid is predominantly glycerol.

Advantages reported for CF-FAB are that the absolute abundances of background ions are lower and that the abundances of analyte ions are inherently higher for CF-FAB than for static FAB. If the film in CF-FAB is predominately glycerol, however, then the absolute abundances of the background ions should be approximately equal for each system. Unfortunately, almost all of the published CF-FAB spectra show only relative abundances. An exception is the published CF-FAB spectrum of $500 \mathrm{fmol}$ of the peptide angiotensin II, which is compared to the static FAB spertrum (see Figure 1.21 in ref 65). Moreover, for such low levels of peptides, the suppression effects resulting from surface activity are expected to be minimal (see Table 4 and compare the absolute abundances of the background ions in Figure 11). The spectra in ref 65 show that, at least at the mass region of the peptide, the absolute abundances of the background ions from dynamic and static $\mathrm{FAB}$ are, indeed, the same $\left(\sim 1 \times 10^{3}\right.$ arbitrary units). The signal-to-noise ratio $(\mathrm{S} / \mathrm{N})$ for the CF-FAB spectrum is higher than that for the static FAB spectrum not because the background ions are less abundant, but rather because the analyte ions are more abundant. The increased abundances of the analyte ions are not inherent, however, to CF-FAB, but can be attributed in large part to the volume of the matrix, which for the thin film in CF-FAB is small and results in a high analyte concentration in the matrix. Recently we showed that reducing the volume of matrix used for static FAB increases the $S / N$, and the use of a thin film of matrix was an important factor in achieving a very luw (picogram) detection limit for the herbicide atrazine [17].

\section{Conclusions}

Many background ions in FAB or LSIMS previously attributed to radiation damage have been identified. The products are of four types: (1) matrix-derived free radical coupling products, (2) their thermallike decomposition products, (3) small molecules resulting from $\mathrm{H}$ atom transfer and $\mathrm{H}$ atom abstraction from the individual free radicals, and (4) aggregates, both noncovalent and covalent, of the matrix with the products

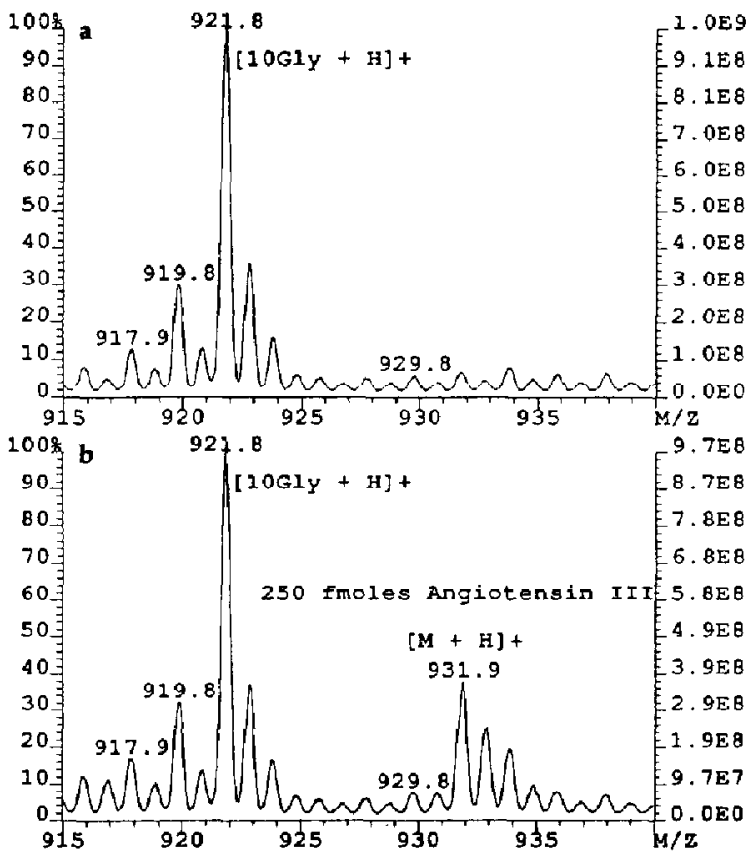

Figure 11. Partial continuous-flow liquid secondary ion mass spectrum of (a) the blank region of background ions and (b) 250 fmol of the peptide angiotensin III. The axis on the right is the absolute abundance.

in 1 through 3. Evidence for the formation of these species romes from acrurate mass measurements and tandem mass spectra.

Because the bombardment products are not preformed, they are not preformed ions, and, thus, they are ionized subsequently by chemical reactions initiated by the impact event. For low-level analyses, there will be minimal suppression of matrix chemistry because surface coverage by the analyte is incomplete. Thus, it is likely that, with current source designs, the matrix chemistry discussed here will prevent instrument detection limits from being achieved in mass spectrometry and MS/MS determination for which $F A B$ is used for ionization.

\section{Supplementary Material}

Supplementary material for this article is available in photocopy form from the office of the Editor-in-Chief (see front of journal for address). Requests must include complete title of article, names of authors, issue date, and page numbers. The supplementary material is a more complete list of positive ions of lower abundance than those in Table 1.

\section{Acknowledgment}

This work was supported by the U.S. National Science Foundation (Grant nos. CHE-9017250 and DIR-9017262). 


\section{References}

1. Honig, R. E. J. Appl. Phys. 1958, $29,549$.

2. McHugh, J. A.; Sheffield, J. J. Appl. Phys. 1964, 35, 512.

3. Benninghoven, A. Z. Naturforsch 1969, $24 a, 859$.

4. Benninghoven, A. Surface Sci. 1973, 35, 427.

5. Benninghoven, A.; Jaspers, D.; Sichtermann, W. Appl. Phys. 1976, 11, 35.

6. Benninghoven, A.; Sichtermann, W. Anal. Chem. 1978, 50, 1180.

7. Day, R. J.; Unger, S. E; Cooks, R. G. Anal. Chem. 1980, 52, $557 \mathrm{~A}$.

8. Barber, M.; Bordoli, R. S.; Sedgewick, R. D.; Tyler, A. N. I. C. S. Chem. Comm. 1981, 325.

9. Barber, M.; Bordoli, R. S.; Sedgewick, R. D.; Tyler, A. N. Nature 1981, 293, 270.

10. Barber, M.; Bordoli, R. S.; Elliot, G. J.; Sedgewick. R. D.; Tyler, A. N. Anal. Chem. 1982, 54,645A.

11. Aberth, W.; Straub, K. M.; Burlingame, A. L. Anal. Chem. 1982, 54, 2029.

12. Falick, A. M.; Wang, G. H.; Walls, F. C. Anal. Chem. 1986, 58, 1308.

13. McEwen, C. N.; Hass, J. R. Anal. Chem. 1985, $57,890$.

14. Green, B. N. Org. Mass Spectrom. 1992, 27, 67.

15. Boerboom, A. J. H. Rapid Commun. Mass Spectrom. 1988, 2 , 260.

16. Light, K. J.; Allison, J. I. Am. Soc. Mass Spectrom. 1990, 1, 455.

17. Caldwell, K. A.; Ramanujam, V. M. S.: Cai, Z; Gross, M. L. Anal. Chem. 1993, 65, 2372.

18. Caldwell, K. A.; Gross, M. L. Proceedirigs of the $39 t h$ Aruual ASMS Conference on Mass Spectrometry and Allied Topics; Nashville, TN, May 19-24, 1991.

19. Busch, K. L.; Glish, G. L.: McLuckey, S. A. Mass Spectrometry/Mass Spectrometry; VCH: New York, 1988.

20. Ligon, W. V.; Dorn, S. B. Int. J. Mass Spectrom. Ion Processes $1984,57,75$

21. Aberth, W. H.; Burlingame, A. L. Anal. Chem. 1988, 60, 1426.

22. Gross, M. L.; Chess, E. K.; Lyon, P. A.; Crow, F. W.; Evans, S.; Tudge, H. Int. I. Mass Spectrom. Ion Phys. 1982, 42, 243.

23. Gross, M. L. In Methods in Enzymology, Mass Spectrometry, vol. 193; McCloskey, J. A., Ed.; Academic: New York, 1990; pP 131-153.

24. Field, F. H. J. Phys. Chem. 1982, 86, 5115.

25. Keough, T.; Ezra, F. S.; Russell, A. F.; Pryne, J. D. Org. Mass Spectrom. 1987, 22, 241.

26. Caldwell, K. A.; Gross, M. L. Presented at the 12th International Conference on Mass Spectrometry, Amsterdam, Holland, August 26-30, 1991.

27. Sunner, J.; Morales, A.; Kebarle, P. Anal Chem. 1988, $60,98$.

28. Sunner, J.; Morales, A.; Kebarle, P. Int. J. Mass Spectrom. Ion Phys. 1988, 86, 169 .

29. Todd, P. J. Org. Mass Spectrom. 1988, $23,419$.

30. Sunner, J.; Ikonomou, M. G.; Kebarle, P. Int. J. Mass Spectrom. Ion Processes 1988, 82, 221.

31. Standing, K. G.; Chait, B. T.; Ens, W.; McIntosh, G.; Beavis, R. Nucl. Instrum. Methods 1982, 198, 33.

32. Sigmund, P. Nucl. Instrum. Methods 1987, B27, 1; Fenselau, C.; Cotter, R. J. Chem. Rev. 1987, 87, 501.

33. Pachuta, S. J.; Cooks, R. G. Chem. Rev. 1987, 87, 647.
34. Katz, R. N.; Chaudary, T.; Field, F. H. Int. J. Mass Spectrom. Ion Phys. 1987, 78, 85.

35. Sunner, J.; Morales, A.; Kebarle, P. Int. J. Mass Spectrom. Ion Process 1989, 87, 287.

36. Neta, P. Advances in Physical Organic Chemistry 1976, 12, 223.

37. Wong, S. S.; Rällgen, F. W.; Manz, I.; Przybylski, M. Binnted. Mass Spectrom. 1985, 12, 43.

38. Sethi, S. K.; Nelson, C. C.: McCloskey, J. A. Anal. Chem. 1984, $56,1975$.

39. Siegel, M. M.; McGahren, W. J.; Ellestad, G. A. In Mass Spectrometry in the Analysis of Large Molecules; McNeal, C. J., Ed.; Wiley: New York, 1986; pp 207-212.

40. Williams, D. H.; Findeis, A. F.; Naylor. S.; Gibson, B. W. J. Am. Chem. Soc. 1987, 109, 1980.

41. McLuckey, S. A.; Cameron, D.; Cooks, R. G. J. Am. Chem. Soc. 1981, 103, 1313.

42. Weinkam, R. J. J. Org. Chem. 1978, 43, 2581.

43. Raftery, M. J.; Bowie, J. H.; Sheldon, J. C. J. Chem. Soc. Perkins Trans. II 1988, 563.

44. Todd, P. J.; Groenwold, G. S. Anal. Chem. 1986, 58, 895.

45. Kilpatrick, G.; Lewis, I. A. S.; Smith, J. F. Biomed. Eno. Mass Spectrom. 1987, 14, 155.

46. Aberth, W. Anal. Chem. 1986, 58, 1221.

47. Kerr, A. J. In Free Radicals, Vol. 1; Kochi, J. K., Ed.; Wiley: New York, 1973; chapter 1.

48. Segur, J. B. In Glycerol; Miner, C. S.; Dalton, N. N., Eds.; Reinhold: New York, 1953; chapter 8.

49. Lehmann, W. D.; Kessler, M.; König. W. A. Biomed. Mass Spectrom. 1984, 11, 217.

50. Johnstone, R. A. W.; Wilby, A. H. Int. J. Mass Spectrom. Ion Processes 1989, 89, 249.

51. Kebarle, P.; Godbole, E. W. J. Chem. Phys. 1963, 39, 1131.

52. Kebarle, P. Ann. Rev. Phys. Chem. 1977, 28, 445.

53. Castleman, Jr., A. W.; Keesee, R. G. Chem. Rev. 1986, 86, 589.

54. Castleman, Jr., A. W. J. Cluster Sci. 1990, 1, 3.

55. Blakley, C. R.; Vestal, M. L. Anal. Chem. 1983, 55, 750.

56. DePauw, E. Mass Spectrom. Rev. 1986, 5, 191.

57. Vékey, K.; Colombo, L.; Zerilli, L. F.; Székely, G. Rapid Commun. Mass Spectrom. 1991, 5, 1.

58. Cox, K. A.; Williams, J. D.; Cooks, R. G.; Kaiser, Jr., R. E. Biol. Mass Spectrom. 1992, 21, 226.

59. Karas, M.; Bachmann, D.; Bahr, U.; Hillenkamp, F. Int. J. Mass Spectrom. Ion Processes 1987, 78, 53.

60. Karas, M.; Ingendoh, A.; Bahr, U.; Hillenkamp, F. Biomed. Env. Mass Spectrom. 1989, 18, 841.

61. Zhao, S.; Somayajula, K. V.; Sharkey, A. G.; Hercules, D. M.; Hillenkamp, F.; Karas, M.; Ingendoh, A. Anal. Chem. 1991, 63, 450.

62. Tuinman, A. A.; Cook, K. D. I. Am. Soc. Mass Spectrom. 1992, 3,318 .

63. Faull, K. F.; Barchas, J. D.; Kenyon, C. N.; Goodley, P. C. Int J. Mass Spectrom. Ion Phys. 1983, 46, 347.

64. Mackitchie, F. Chemistry at interfaces; Academic: San Diego, 1990.

65. Continuous-Flow Fast Atom Bombardment Mass Spectrometry; Caprioli, R. M., Ed.; Wiley: New York, 1990.

66. Heine, C. E.; Holland, J. F; Watson, J. T. Anal. Chem. 1989, 61, 2674. 